\title{
Divergent encoding of active avoidance behavior in corticostriatal and corticolimbic
} projections

Bridget L. Kajs ${ }^{1, *}$, Adrienne C. Loewke ${ }^{1, *}$, Jeffrey M. Dorsch ${ }^{1}$, Leah T. Vinson², Lisa A. Gunaydin ${ }^{1,3, \dagger}$

1 Department of Psychiatry and Behavioral Sciences, University of California San Francisco, San Francisco CA

2 Department of Neurology, University of California San Francisco, San Francisco CA

${ }^{3}$ Kavli Institute for Fundamental Neuroscience, University of California San Francisco, San Francisco CA

* These authors contributed equally.

† Corresponding author (lisa.gunaydin@ucsf.edu) 


\section{ABSTRACT}

Active avoidance behavior, in which an animal performs an action to avoid a stressor, is crucial for survival and may provide insight into avoidance behaviors seen in anxiety disorders. Active avoidance requires the dorsomedial prefrontal cortex (dmPFC), which is thought to regulate avoidance via downstream projections to the striatum and amygdala. However, the endogenous activity of projection-defined dmPFC subpopulations during active avoidance learning remains unexplored. Here we utilized fiber photometry to record from the dmPFC and its downstream projections to the dorsomedial striatum (DMS) and the basolateral amygdala (BLA) during active avoidance learning in mice. We examined neural activity during conditioned stimulus (CS) presentations, active avoidance, and cued freezing. Both prefrontal projections showed learningrelated increases in activity during CS onset throughout active avoidance training. The dmPFC as a whole showed increased activity during avoidance and decreased activity during cued freezing. Finally, dmPFC-DMS and dmPFC-BLA projections showed divergent encoding of active avoidance behavior, with the dmPFC-DMS projection showing increased activity and the dmPFCBLA showing decreased activity during active avoidance. Our results identify differential prefrontal encoding of active and passive coping behaviors in the same behavioral paradigm and demonstrate divergent encoding of active avoidance in projection-specific dmPFC subpopulations. 


\section{INTRODUCTION}

Active avoidance is a behavioral coping strategy in which an organism performs an action to avoid a stressor and can be adaptively enacted to evade danger and ensure survival. However, active avoidance can become maladaptive when used in excess or in response to overexaggerated perceived threats as seen in anxiety disorders. Despite its high clinical relevance, our understanding of the neurobiological basis of active avoidance has lagged far behind other behaviors relevant to anxiety disorders such as approach-avoidance decision making or fear learning (LeDoux et al., 2017). The dorsomedial prefrontal cortex (dmPFC) is an attractive candidate to explore in the context of active avoidance given its clear ties to anxiety disorder pathophysiology (Holzschneider \& Mulert, 2011; Rauch \& Shin, 2002) and avoidance behavior in humans (Collins et al., 2014; Delgado et al., 2009) as well as clinically relevant behaviors in rodents (Giustino \& Maren, 2015; Tovote et al., 2015). In non-psychiatric populations, dmPFC activity is associated with active avoidance learning (Collins et al., 2014) while in post-traumatic stress disorder (PTSD) patients, dmPFC activation during fear extinction positively correlates with patients' avoidance symptoms (Sripada et al., 2013). In rodents, dmPFC plays a crucial role in associative fear learning (Adhikari et al., 2015; Corcoran \& Quirk, 2007; Courtin et al., 2014; Dejean et al., 2016; Fenton et al., 2014; Giustino \& Maren, 2015; Herry \& Johansen, 2014; Klavir et al., 2017; Marek et al., 2018; Meyer et al., 2019; Sharpe \& Killcross, 2014; Sierra-Mercado et al., 2011; Sotres-Bayon et al., 2012; Tovote et al., 2015) and instrumental action-outcome learning (Gourley \& Taylor, 2016; Grace et al., 2007; Peters et al., 2005; Pinto \& Dan, 2015), both of which are components of active avoidance behavior. Studies have directly demonstrated the importance of the dmPFC for a variety of avoidance behaviors including real time and conditioned place avoidance (Huang et al., 2020; Lee et al., 2014; Vander Weele et al., 2018), inhibitory avoidance (Garrido et al., 2012; Ito \& Morozov, 2019; Izquierdo et al., 2007; Torres-García et al., 2017; Zhang et al., 2011), approach-avoidance decision making (Friedman et al., 2015; Loewke 
25 et al., 2021), and active avoidance (Beck et al., 2014; Bravo-Rivera et al., 2014; Capuzzo \&

26 Floresco, 2020; Diehl et al., 2018, 2020). One recent study using the platform-mediated active

27 avoidance task showed that suppression of dmPFC activity is associated with avoidance learning

28 (Diehl et al., 2018). Another study using a discriminative two-way active avoidance paradigm

29 found that dmPFC population activity alone could be used to decode conditioned stimulus (CS)

30 identity between a conditioned stimulus that predicted shock and led to robust avoidance behavior

$31(\mathrm{CS}+)$ and a conditioned stimulus that did not predict to shock and did not lead to avoidance (CS-

32 ) (Jercog et al., 2021). In these studies, task-relevant neural activity in the dmPFC during active

33 avoidance has only been examined on the final day of active avoidance training after learning has

34 already occurred. To our knowledge, no studies have thoroughly examined dmPFC activity

35 throughout avoidance learning. Investigating how task-relevant signals in the dmPFC develop in

36 real time across days of learning could help determine whether the dmPFC is preferentially

37 recruited during certain stages of learning or whether task-relevant dmPFC activity requires

38 consolidation across days.

39 Further dissecting the dmPFC into subpopulations based on their projection target may also yield

40 more refined insights into the nuanced and varied roles of the dmPFC in active avoidance

41 behavior. One downstream target of the dmPFC that has been consistently tied to active

42 avoidance behavior is the basolateral amygdala (BLA) (Amorapanth et al., 2000; Bravo-Rivera et

43 al., 2014; Choi et al., 2010; Darvas et al., 2011; Diehl et al., 2020; Killcross et al., 1997; Kyriazi et

44 al., 2018; Lázaro-Muñoz et al., 2010; Maren et al., 1991; Poremba \& Gabriel, 1999). The BLA has

45 subpopulations of cells that specifically encode successful active avoidance behavior (Kyriazi et

46 al., 2018), and inactivating the BLA impairs platform-mediated active avoidance behavior (Bravo-

47 Rivera et al., 2014). Additionally, the dmPFC-BLA projection has been directly tied to active

48 avoidance, as optogenetically stimulating or inhibiting this projection bidirectionally affects

49 platform-mediated active avoidance behavior (Diehl et al., 2020). However, despite these 
optogenetic studies suggesting a causal role of this projection in avoidance behavior, no studies

51 have directly recorded the endogenous activity in this projection subpopulation during active

52 avoidance learning or expression. The task-relevant information of the dmPFC-BLA projection in

53 active avoidance could be multifold. dmPFC-BLA projections could signal crucial information

54 about the cue-shock association, as the BLA receives associative information that has converged

55 upstream in the lateral amygdala (LA) (Duvarci \& Pare, 2014; Tovote et al., 2015). dmPFC-BLA

56 projections may also directly impact behavioral output by amplifying avoidance information sent

57 to the nucleus accumbens (LeDoux et al., 2017; Ramirez et al., 2015) and suppressing fearful

58 freezing information sent to the central amygdala (LeDoux et al., 2017; Terburg et al., 2018).

59 However, it remains unknown how the real-time neural dynamics in this projection encode active

60 avoidance, which would require projection-specific recording of dmPFC-BLA projection neurons

61 during avoidance learning and expression.

62 While corticolimbic projections have been heavily studied in the context of fear conditioning,

63 recent evidence suggests that corticostriatal projections also play a key role in avoidance behavior

64 (Friedman et al., 2015; Loewke et al., 2021). Human fMRI studies have implicated both the dorsal

65 and ventral striatum in active avoidance behavior (Boeke et al., 2017; Collins et al., 2014; Delgado

66 et al., 2009). However, while the ventral striatum has been more thoroughly studied in rodent

67 models (Bravo-Rivera et al., 2014, 2015; Darvas et al., 2011; Gentry et al., 2016; Oleson et al.,

68 2012; Piantadosi et al., 2018; Ramirez et al., 2015; Rodriguez-Romaguera et al., 2016; Stelly et

69 al., 2019; Wenzel et al., 2018), there has been less exploration into the role of the dorsal striatum

70 in active avoidance (Boschen et al., 2011; Dombrowski et al., 2013; Wendler et al., 2014;

71 Wietzikoski et al., 2012). dmPFC projections to the dorsal striatum, especially the dorsomedial

72 subregion (DMS), are uniquely positioned to play a crucial role in active avoidance behavior given

73 their importance in goal-directed behavior (Balleine \& O'Doherty, 2010; Gremel \& Costa, 2013;

74 Hart, Bradfield, \& Balleine, 2018; Hart, Bradfield, Fok, et al., 2018; Pitts et al., 2018) and 
75 approach-avoidance decision making (Friedman et al., 2015; Loewke et al., 2021). Additionally in

76 humans, the degree of coupling between the caudate (the human homologue of the DMS) and

77 the medial prefrontal cortex (mPFC) positively correlates with successful active avoidance

78 performance with greater coupling predicting better performance (Collins et al., 2014). The

79 dmPFC-DMS projection could hold task-relevant information regarding action-outcome

80 contingencies necessary for goal-directed behavior (Balleine \& O’Doherty, 2010; Yin \& Knowlton,

81 2006). As the dmPFC-DMS projection directly interfaces with the striatum, this projection could

82 also carry crucial information for avoidance initiation through movement-promoting pathways

83 (Kravitz \& Kreitzer, 2012; Redgrave et al., 2010). Despite promising initial evidence and strong

84 rationale for its involvement, the dmPFC-DMS projection has remained completely unexplored in

85 rodent models of active avoidance.

86 In this study, we utilize fiber photometry in combination with retrograde viral targeting strategies

87 to examine the activity of the dmPFC and its projections to the DMS and the BLA during learning

88 and expression in a cued active avoidance task. We identified task-relevant neural activity in

89 response to CS onset as well as clinically relevant behaviors such as avoidance and freezing. We

90 find that dmPFC and both of these downstream projections show learning-related increases in

91 activity at CS onset. However, encoding by these projections diverges during avoidance onset,

92 where we find increased activity in the dmPFC-DMS projection and decreased activity in the

93 dmPFC-BLA projection. Finally, we identify decreases in dmPFC activity that correspond to

94 freezing bouts. Overall, our results suggest that dmPFC and its projections to DMS and BLA

95 contain task-relevant information and that the dmPFC-DMS and dmPFC-BLA may play distinct

96 yet complementary roles in successful enactment of active avoidance behavior. 


\section{RESULTS}

\section{dmPFC shows learning related increases in activity at CS onset}

98 To record the endogenous activity of excitatory dmPFC neurons during avoidance learning, we

99 utilized a virally-expressed calcium indicator (GCaMP) and fiber photometry to record changes in

100 GCaMP fluorescence in the dmPFC, which acted as a proxy for changes in neural activity (Figure

101 1A, Supplemental Figure 1). Mice were trained for five days on a cued two-way active avoidance

102 behavioral paradigm (Figure 1B). A white light underneath the shock floor where the animal was

103 present acted as a conditioned stimulus (CS) and signaled impending shock on that side of the

104 two-chamber apparatus. Throughout training, animals learned to successfully avoid the

105 impending shock by shuttling from the lit chamber to the unlit chamber during the CS-only period.

106 Animals were trained until they successfully avoided the shock on $80 \%$ of all trials, which occurred

107 by day 5 of training (Figure 1C). Average avoidance latency was between 4-6 seconds and

108 decreased across training. Avoidance latencies also became more stereotyped as evidenced by

109 a change in the shape of the avoidance latency distribution from a broad non-specific curve on

110 day 1 to a narrower distribution on day 5 (Figure 1D). To uncover task-relevant neural activity in

111 the dmPFC during active avoidance learning, we first examined heatmaps of the average change

112 in calcium signal in the dmPFC for each trial during the CS-only period (first 10 seconds of the

113 CS before the shock occurred) (Figure 1E). We saw a rapid peak in fluorescence at CS onset on

114 day 1 that occurred on most but not all trials and became more consistent throughout training. In

115 addition to this rapid CS response, we also observed a sustained increase in fluorescence across

116 the 10 second CS-only period that appeared to develop across learning, as it was consistently

117 present on days 3 and 5 but not day 1 . While these heatmaps provided initial insight that there

118 were task-relevant changes in the dmPFC during active avoidance learning, it was unclear

119 whether these changes in calcium signal were a response to the CS itself and/or represented

120 behaviors such as avoidance or freezing. In order to isolate CS onset responses, we created a 
121 perievent time histogram (PETH) of z-scored changes in dmPFC calcium signal during the first 122 second of the CS presentation as the majority of avoidance movements (>90\%) occurred after 123 this time window (Figure 1F). We found that the dmPFC showed a sharp increase in fluorescence 124 during the first second of CS onset compared to the baseline period; this effect was significant on 125 all training days. However, the magnitude of the increase in fluorescence significantly increased 126 across days, with the smallest CS-related change in fluorescence occurring on day 1 and the 127 largest CS-related change in fluorescence occurring on day 5 (Figure 1G, Two-way ANOVA, 128 Training Day $x$ Task Period $p<0.0001$, Training Day $p<0.0001$, Task Period $p<0.0001$; Sidak's 129 Multiple Comparisons Test, Day 1 Baseline vs Day 3 Baseline $p=0.9949$, Day 1 Baseline vs Day 1305 Baseline $p=0.9684$, Day 1 Baseline vs Day 1 CS $p<0.0001$, Day 1 CS vs Day 3 CS $p<$ 1310.0001 , Day 1 CS vs Day 5 CS p < 0.0001, Day 3 Baseline vs Day 5 Baseline $p>0.9999$, Day 3 132 Baseline vs Day 3 CS $p<0.0001$, Day 3 CS vs Day 5 CS $p<0.0001$, Day 5 Baseline vs Day 5 133 CS $p<0.0001 ; N=10$ mice, $n=300$ trials). There were no significant within-day differences in 134 the amplitude of the dmPFC calcium signal when comparing dmPFC fluorescence during the 15 135 first trials to the last 15 trials within a given training day (Supplemental Figure 2). We also found 136 no differences in calcium signal between successful and unsuccessful trials during the first second 137 after CS onset; however, there were statistically significant differences during the later part of the 138 PETH during the time window in which avoidance actions occur (Supplemental Figure 3). Taken 139 together, these data suggest that there are learning-related increases in neural activity in the 140 dmPFC during CS onset that become amplified across active avoidance learning. 
A

Fiber Photometry Recording

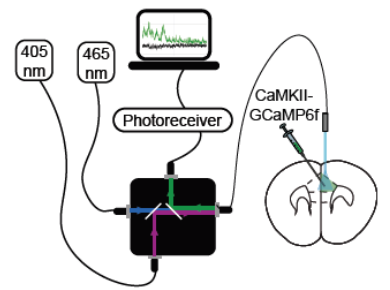

C

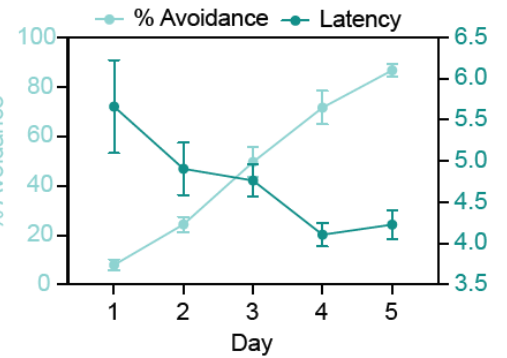

B

\section{Kajs et al. Figure 1}

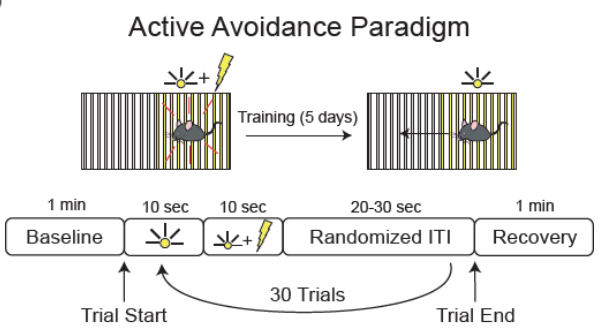

D

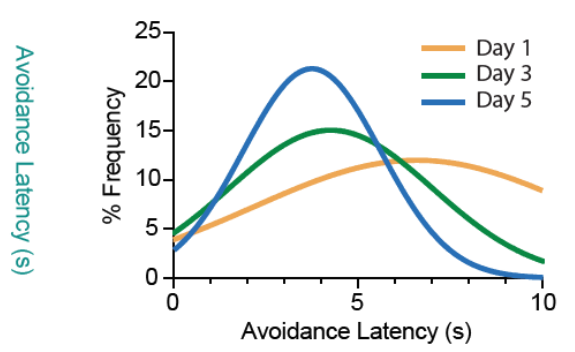

$E$

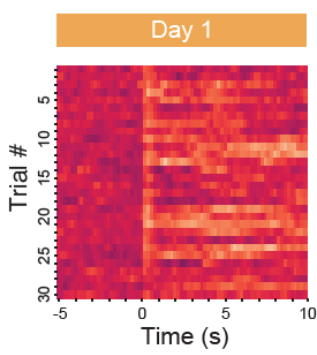

F

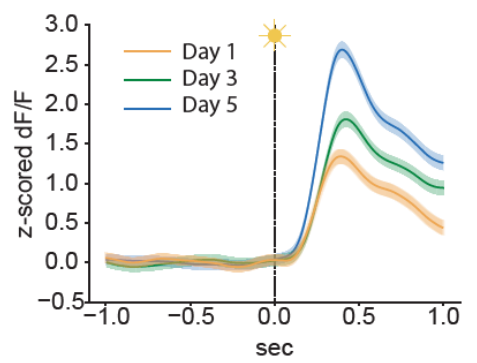

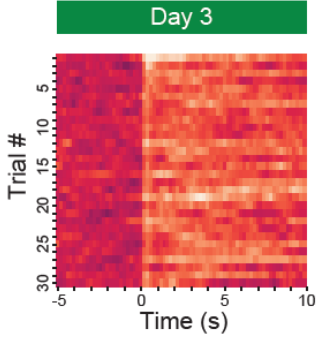

G
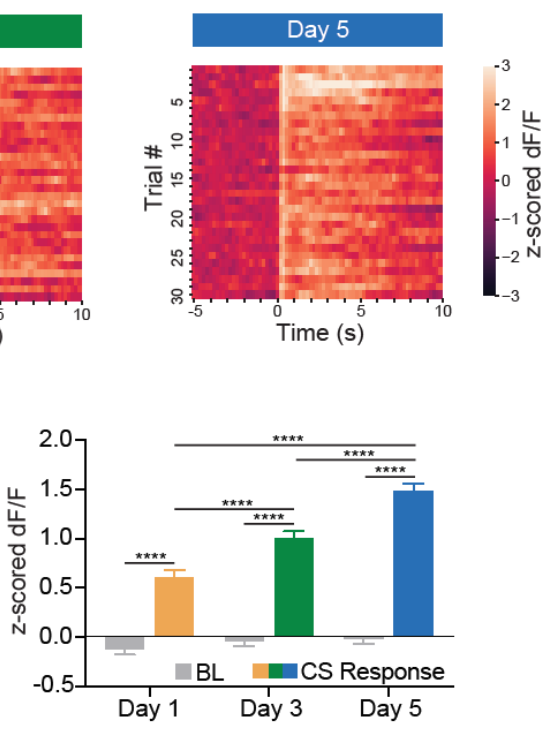

Figure 1. dmPFC shows learning-related increases in activity at CS onset during active avoidance learning. (A) Fiber photometry recording of dmPFC pyramidal neurons expressing GCaMP6f. (B) Behavioral schematic for active avoidance paradigm. (C) Average percent successful avoidance increased while avoidance latency decreased across training days. (D) Avoidance latency distribution shows avoidance latencies become shorter and more stereotyped across training. (E) Heatmaps of average change in calcium signal (z-scored dF/F) for each of the 30 trials presented in order from the first to the last trial for Day 1 (left), Day 3 (middle), and Day 5 (right). Heatmaps are aligned to CS onset (time zero) and show the total 10 second CS only period. dmPFC shows increased calcium signal at CS onset that becomes more consistent and sustained with training. (F) Perievent time histogram (PETH) showing increases in dmPFC calcium signal following CS onset. Orange line, mean \pm standard error of the mean (SEM) for Day 1; green line, mean \pm SEM for Day 3; blue line, mean \pm SEM for Day 5. (G) Quantification of CS onset PETH shows calcium signal is significantly higher during the CS period $(0$ to $1 \mathrm{~s})$ compared to the baseline period $(-1$ to $0 \mathrm{~s})$ for all days. ${ }^{* * *} p \leq 0.0001$. 
142 We next sought to examine dmPFC neural activity that corresponded to active avoidance and

143 freezing behaviors that occurred later during the CS presentation. We investigated freezing

144 behavior in addition to avoidance as freezing represents an alternative coping strategy that

145 animals utilize early in learning before active coping strategies such as avoidance have been

146 learned. The number of successful avoidances significantly increased across learning (Figure

147 2A-B, Repeated Measures One-way ANOVA p < 0.0001; Sidak's Multiple Comparisons Test, Day

1481 vs Day $3 p=0.0002$, Day 1 vs Day $5 p<0.0001$, Day 3 vs Day $5 p<0.0001 ; N=10$ mice).

149 When aligning the dmPFC calcium signal to avoidance onset on day 5 (Figure 2C), we found a

150 statistically significant increase in fluorescence during the avoidance period compared to the

151 baseline period (Figure 2D, Repeated Measures One-Way ANOVA $p<0.0001$; Tukey's Multiple

152 Comparisons Test, Baseline vs Pre Avoid $p<0.0001$, Baseline vs Avoid $p<0.0001$, Baseline vs

153 Post Avoid $p<0.0001$, Pre Avoid vs Avoid $p<0.0001$, Pre Avoid vs Post Avoid $p=0.6062$, Avoid

154 vs Post Avoid $p<0.0001 ; N=10$ mice, $n=253$ trials). To rule out the possibility that these neural

155 activity changes during avoidance onset in the dmPFC could be purely movement-related, we

156 compared calcium signal during non-avoidance movements in the intertrial interval (ITI) period to

157 avoidance movements of a similar velocity or duration from the same recording day. We found

158 significantly increased fluorescence during avoidance movements compared to ITI movements

159 during the time period where movements are initiated, suggesting that the increase in calcium

160 signal during avoidance movements was not purely movement-related (Supplemental Figure 4).

161 To further characterize the nature of the neural activity changes during avoidance, we created

162 heatmaps of calcium activity on all individual trials aligned to avoidance onset and sorted them

163 from shortest to longest avoidance latency (Figure 2E). In this heatmap, we observed a consistent

164 time-locked peak in fluorescence that corresponded to avoidance onset. We also saw a sharp

165 moving peak of fluorescence curving leftward that likely represented the increase in calcium signal 
at CS onset. These data suggest that the dmPFC separately encodes both the CS onset and

167 avoidance onset through distinct increases in neural activity.

168 In contrast to avoidance, the amount of freezing during the CS-only period (cued freezing) 169 significantly decreased across learning (Figure 2F-G, Repeated Measures One-way ANOVA p = 170 0.0045; Sidak's Multiple Comparisons Test, Day 1 vs Day $3 p=0.4807$, Day 1 vs Day $5 p=$ 1710.0024 , Day 3 vs Day $5 p=0.023 ; N=10$ mice). When we generated a PETH of dmPFC calcium 172 activity aligned to freezing onset on day 1 for all cued freezing bouts with a 1 second minimum 173 duration (Figure $\mathbf{2 H}$ ), we found a statistically significant decrease in fluorescence during the 174 freezing period compared to the baseline period (Figure 2l, Paired t-test $p<0.0001 ; N=10$ mice, $175 \mathrm{n}=246$ trials). When examining a heatmap of calcium activity on all individual trials aligned to 176 freezing onset and sorted by shortest to longest freezing bout duration, we saw a dip in 177 fluorescence at freezing onset that increased in duration with longer freezing bouts (Figure 2J).

178 This suggested that the duration of the decrease in dmPFC calcium activity during freezing 179 corresponded to the duration of the freezing bout length, providing further evidence that the dip 180 in fluorescence was tightly time-locked with freezing behavior. Overall, our results suggest that 181 the dmPFC shows opposing patterns of activity during avoidance and freezing and that these 182 patterns of activity are distinct from the neural activity observed during CS onset. 

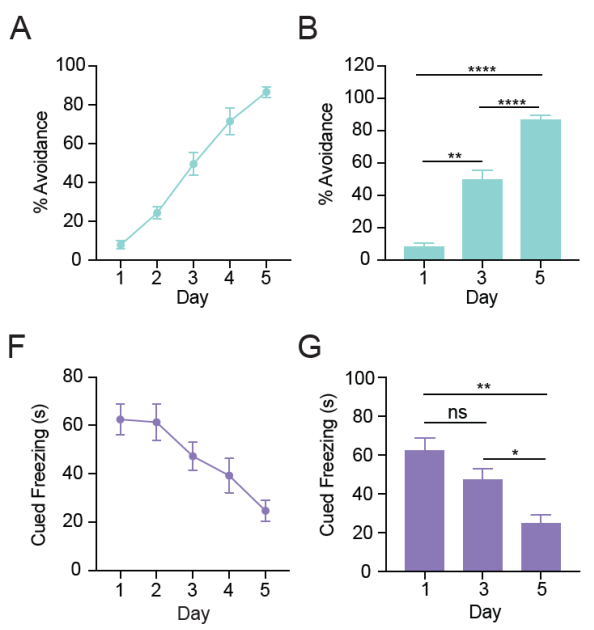

C

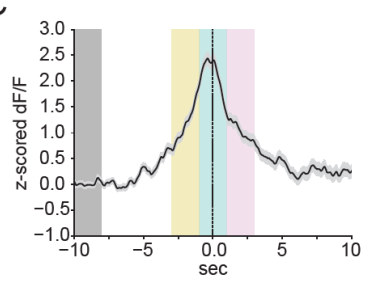

$\mathrm{H}$

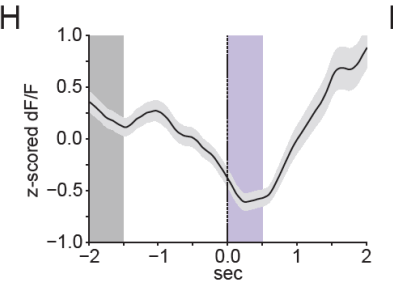

D

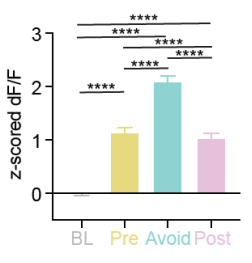

I

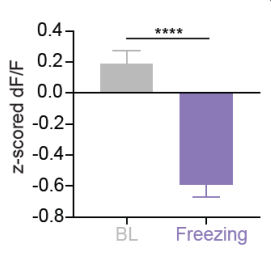

Kajs et al. Figure 2

$\mathrm{E}$

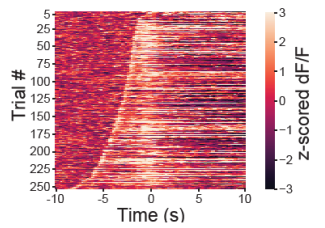

$\mathrm{J}$

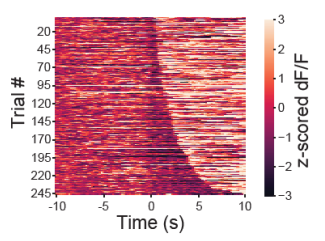

Figure 2. dmPFC shows opposing patterns of activity during active avoidance and cued freezing behavior. (A) Percent successful avoidance across training days. (B) Quantification of percent successful avoidance shows animals significantly increase avoidance across training. (C) PETH shows an increase in calcium signal at avoidance onset. Line with shading represents mean \pm SEM. Grey box, baseline period (BL); yellow box, pre avoidance period (Pre); teal box, avoidance period (Avoid); pink box, post avoidance period (Post). (D) Quantification of avoidance PETH reveals significantly increased calcium signal in pre avoid (-3 to $-1 \mathrm{~s}$ ), avoid (-1 to $1 \mathrm{~s}$ ), and post avoid (1 to $3 \mathrm{~s}$ ) periods compared to the baseline period (-10 to $-8 \mathrm{~s})$. (E) Heatmap of change in calcium signal for individual avoidance trials aligned to avoidance onset and sorted from shortest to longest avoidance latency. Heatmap shows distinct increases in calcium signal at CS onset (slope curving leftward) and avoidance onset (time zero). (F) Percent freezing during the CS only period (cued freezing) across training days. (G) Quantification of percent cued freezing shows that animals significantly decrease cued freezing across training. (H) PETH shows decrease in calcium signal at freezing onset. Line with shading represents mean \pm SEM. Grey box, baseline period (BL); Purple box, freezing period (Freezing). (I) Quantification of freezing PETH shows significant decrease in calcium signal during the freezing period (0-0.5 s) compared to the baseline period (-2 to $-1.5 \mathrm{~s})$. (J) Heatmap of change in calcium signal during individual freezing bouts aligned to freezing onset and sorted from shortest to longest freezing bout. Heatmap shows dips in calcium signal at freezing onset that increases in length as freezing bout duration increases. $n s=$ not significant, ${ }^{*} p \leq 0.05,{ }^{* *} p \leq 0.01,{ }^{* * *} p \leq 0.0001$. 


\section{CS onset}

We also explored how subpopulations of dmPFC neurons defined by their downstream projection target may diverge in the encoding of active avoidance. To obtain projection-specific fiber photometry recordings from the dmPFC-DMS projection, we used a dual virus retrograde targeting strategy to express GCaMP only in dmPFC neurons projecting to the DMS (Figure 3A,

Supplemental Figure 5). Behavioral results from this cohort revealed that the mice learned to $80 \%$ successful avoidance by day 5 , average avoidance latencies were between 4- 6 seconds, and avoidance latency decreased across training (Figure 3B). To visualize potential task-relevant information within the dmPFC-DMS projection, we examined heatmaps of the average calcium signal change on each trial for the first 10 seconds of the CS (CS-only period) (Figure $3 \mathrm{C}$ ). In the dmPFC-DMS projection, already on the first day of learning we saw a sustained increase in fluorescence during the CS only period, although the start of the signal did not appear clearly time locked to CS onset and the sustained increase did not appear on every trial. However, by day 5 197 of learning the sustained increase in fluorescence in the dmPFC-DMS projection became time locked to CS onset and consistently appeared on every trial. When examining calcium activity during the first second of CS onset in the dmPFC-DMS projection (Figure 3D), we found a 200 significant increase in signal at CS onset compared to baseline on day 5 but not on day 1 . We 201 additionally found that there was a significant difference in calcium signal at CS onset across 202 days, with a larger CS-evoked increase in signal on day 5 compared to day 1, suggesting that 203 there were learning-related changes (Figure 3E, Two-way ANOVA, Training Day x Task Period $204 p=0.0498$, Training Day $p=0.0725$, Task Period $p<0.0001$; Sidak's Multiple Comparisons Test, 205 Day 1 Baseline vs Day 1 CS $p=0.0634$, Day 1 Baseline vs Day 5 Baseline $p>0.9999$, Day 1 CS 206 vs Day 5 CS $p=0.0466$, Day 5 Baseline vs Day 5 CS $p<0.0001 ; N=8$ mice, $n=300$ trials). 
207 We next examined neural activity in the dmPFC-BLA projection during active avoidance learning 208 using the same dual virus retrograde targeting strategy (Figure 3F, Supplemental Figure 5). 209 Behaviorally, we saw similar trends to the dmPFC-DMS projection cohort (Figure 3G). Heatmaps 210 of the average calcium activity change during the first 10 seconds of the CS revealed that the

211 dmPFC-BLA projection did not show clearly organized patterns of fluorescence on the first day of 212 learning. However, by day 5 this projection showed a clear transient increase in fluorescence that 213 was time locked to CS onset and consistently seen across trials (Figure $\mathbf{3 H}$ ). When examining 214 calcium activity in the dmPFC-BLA during the first second of CS onset across learning (Figure $2153 \mathrm{I}$ ), the dmPFC-BLA projection showed no significant differences in signal between the baseline 216 period and CS onset on day 1, but showed significant increases in calcium signal at CS onset 217 compared to the baseline period on day 5. We also found that there was a significant increase in 218 signal at CS onset on day 5 of learning compared to day 1 of learning (Figure 3J, Two-way 219 ANOVA, Training Day $x$ Task Period $p=0.0816$, Training Day $p=0.0411$, Task Period $p<0.0001$; 220 Sidak's Multiple Comparisons Test, Day 1 Baseline vs Day 5 Baseline $p>0.9999$, Day 1 Baseline 221 vs Day 1 CS $p=0.3023$, Day 1 CS vs Day 5 CS $p=0.0442$, Day 5 Baseline vs Day 5 CS $p<$ $2220.0001 ; \mathrm{N}=9$ mice, $\mathrm{n}=300$ trials). Additional analyses examining calcium activity in these 223 projections during successful and unsuccessful trials found that the CS-evoked fluorescence 224 changes during successful trials did not significantly differ from that on unsuccessful trials for 225 either projection (Supplemental Figure 6). Overall, our results suggest that both the dmPFC226 DMS and dmPFC-BLA projections show learning-related increases in neural activity at CS onset 227 during active avoidance learning. 
A

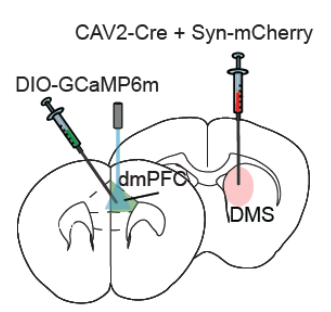

C

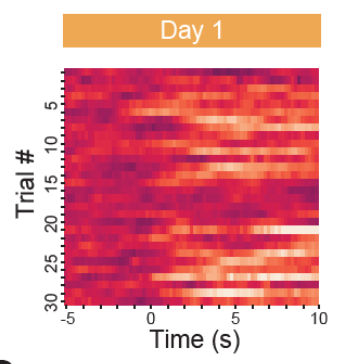

D

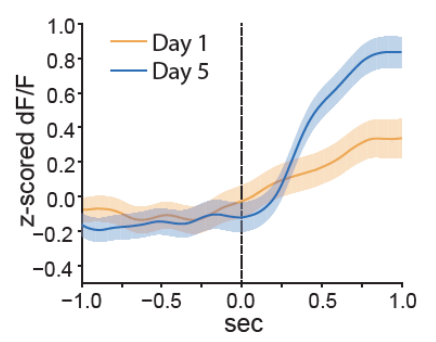

B
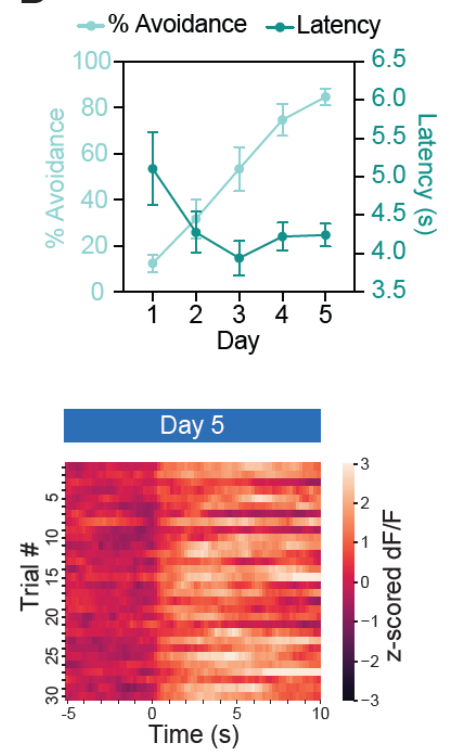

E

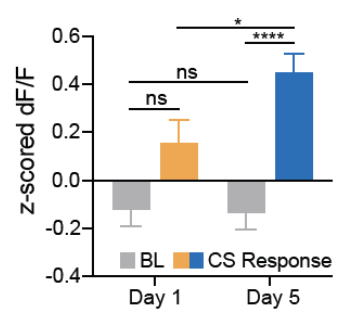

F

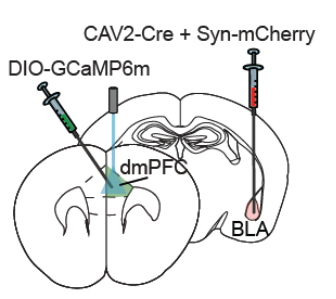

$\mathrm{H}$

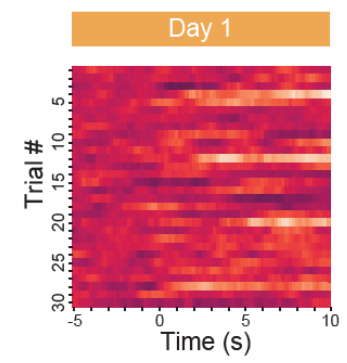

I

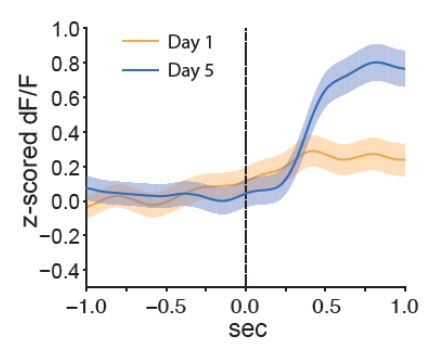

Kajs et al. Figure 3

$\mathrm{G}$
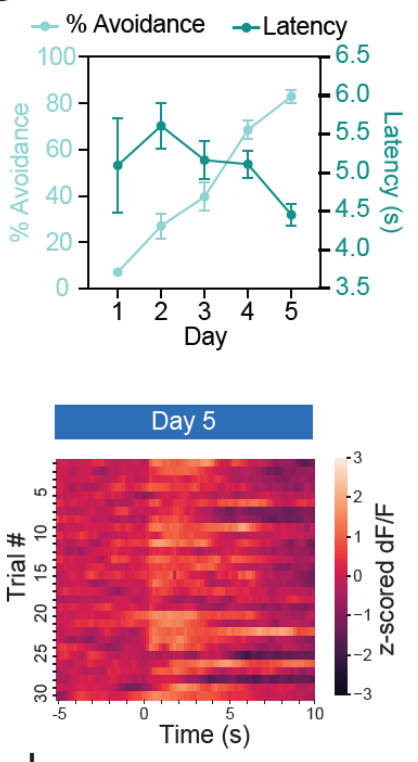

$\mathrm{J}$

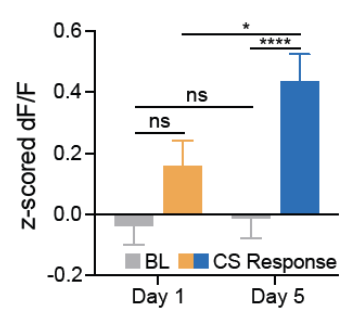

Figure 3. dmPFC-DMS and dmPFC-BLA show similar learning-related increases in activity at CS onset during active avoidance learning. (A) Viral targeting strategy for dmPFC-DMS photometry. (B) Percent avoidance increases while avoidance latency decreases across training in the dmPFC-DMS cohort. (C) Heatmaps of change in calcium signal aligned to CS onset for each of the 30 trials arranged from first to the last trial for Day 1 (left) and Day 5 (right). dmPFC-DMS projection shows sustained increases in calcium signal at CS onset that become more consistent across training. (D) PETH shows increases in signal at CS onset in the dmPFC-DMS projection following training. orange line, mean \pm SEM for Day 1; blue line, mean \pm SEM for Day 5. (E) Quantification of the dmPFC-DMS CS onset PETH shows significant increase in calcium signal during the CS period ( 0 to $1 \mathrm{~s}$ ) compared to the baseline period ( -1 to $0 \mathrm{~s}$ ) for Day 5 , but not Day 1. (F) Viral targeting strategy for dmPFC-BLA photometry. (G) Percent avoidance increases while avoidance latency decreases across training in the dmPFC-BLA cohort. $(H)$ Heatmaps of change in calcium signal aligned to CS onset for each of the 30 trials arranged from first to the last trial for Day 1 (left) and Day 5 (right). dmPFC-BLA projection shows transient increases in calcium signal at CS onset only during later stages of training. (I) PETH shows increases in signal at CS onset in the dmPFC-BLA projection following training. orange line, mean \pm SEM for Day 1; blue line, mean \pm SEM for Day 5. (J) Quantification of the dmPFC-BLA CS onset PETH shows significant increase in calcium signal during the CS period (0 to $1 \mathrm{~s}$ ) compared to the baseline period (-1 to $0 \mathrm{~s}$ ) for Day 5 , but not Day 1. ns $=$ not significant, ${ }^{*} p \leq 0.05,{ }^{* * * *} p$ $\leq 0.0001$. 
230 We were additionally interested in examining projection-specific neural activity during avoidance

231 and freezing behaviors. Both cohorts reached $80 \%$ successful avoidance by day 5 of learning

232 (Figure 4A-C, dmPFC-DMS Paired t-test $p<0.0001$, dmPFC-BLA Paired t-test $p<0.0001$;

233 dmPFC-DMS N = 8 mice, dmPFC-BLA $N=9$ mice). While calcium activity in these two projections

234 was similar upon CS onset, we found a striking contrast in avoidance-related calcium activity

235 between the dmPFC-DMS and dmPFC-BLA projections. In the PETH aligned to avoidance onset,

236 while the dmPFC-DMS projection showed a hill-like increase in fluorescence at avoidance onset,

237 the dmPFC-BLA projection showed a descending slope (Figure 4D). Validating these stark

238 changes, the dmPFC-DMS projection showed a significant increase in signal during the

239 avoidance period compared to the baseline period while the dmPFC-BLA projection showed a

240 significant decrease in signal between the pre-avoidance and post avoidance periods. In addition,

241 the dmPFC-DMS and the dmPFC-BLA calcium signals were distinct from each other as they

242 statistically differed throughout the avoidance and post-avoidance periods (Figure 4E, Two-way

243 ANOVA, Task Period $x$ Projection $p<0.0001$, Task Period $p<0.0001$, Projection $p<0.0001$;

244 Sidak's Multiple Comparisons Test, dmPFC-DMS Baseline vs dmPFC-DMS Avoid $p<0.0001$,

245 dmPFC-BLA Pre Avoid vs dmPFC-BLA Post Avoid $p<0.0001$, dmPFC-DMS Baseline vs dmPFC-

246 BLA Baseline $p>0.9999$, dmPFC-DMS Pre Avoid vs dmPFC-BLA Pre Avoid $p=0.9837$, dmPFC-

247 DMS Avoid vs dmPFC-BLA Avoid $p<0.0001$, dmPFC-DMS Post Avoid vs dmPFC-BLA Post

248 Avoid $\mathrm{p}<0.0001$; dmPFC-DMS $\mathrm{N}=8$ mice, $\mathrm{n}=195$ trials, dmPFC-BLA $\mathrm{N}=9$ mice, $\mathrm{n}=211$

249 trials). Using movements of similar velocity or duration during the ITI period as a control, we found

250 significant differences in fluorescence between the ITI movements compared to avoidance

251 movements, suggesting that the changes in calcium activity in these projections during avoidance

252 onset were not purely movement-related (Supplemental Figure 7). To further characterize the

253 avoidance-related activity seen in these projections, we created heatmaps of calcium activity on 
254 all individual trials aligned to avoidance onset sorted from shortest to longest avoidance latency

255 for each projection (Figure 4F). In the dmPFC-DMS projection avoidance heatmap, we saw an

256 increase in fluorescence that curved leftwards, which corresponded to the start of the CS. This

257 increased fluorescence that occurred at CS onset was sustained through avoidance onset as

258 there were no clear distinctions in signal between when the CS began and when the avoidance

259 began. In contrast, in the dmPFC-BLA projection heatmap, CS onset and avoidance onset were

260 marked by distinct changes in calcium activity. There was a clear increase in fluorescence sloping

261 leftward that corresponded to CS onset, whereas avoidance onset was marked by a time-locked

262 drop in fluorescence.

263 We next examined how the dmPFC-DMS and the dmPFC-BLA projections encoded freezing

264 behavior and found statistically significant decreases in freezing on day 5 compared to day 1 for

265 each projection (Figure 4G-I, dmPFC-DMS Paired t-test $p=0.0484$, dmPFC-BLA Paired t-test $p$

$266=0.0032 ;$ dmPFC-DMS N = 8 mice, dmPFC-BLA N = 9 mice). However, there was no significant

267 difference in signal between the baseline period and the freezing period in the perievent time

268 histograms aligned to freezing onset for each projection (Figure 4J-K, Two-way ANOVA, Task

269 Period $x$ Projection $p=0.9234$, Task Period $p=0.8965$, Projection $p=0.0145$; Sidak's Multiple

270 Comparisons Test, dmPFC-DMS Baseline vs dmPFC-BLA Baseline $p=0.4562$, dmPFC-DMS

271 Baseline vs dmPFC-DMS Freezing $\mathrm{p}>$ 0.9999, dmPFC-BLA Baseline vs dmPFC-BLA Freezing

$272 p>0.9999$, dmPFC-DMS Freezing vs dmPFC-BLA Freezing 0.3624; dmPFC-DMS N = 8 mice, $n$

$273=183$ trials, dmPFC-BLA N = 9 mice, $n=229$ trials). Overall, our results show opposing patterns

274 of activity in the dmPFC-DMS and dmPFC-BLA projection during active avoidance behavior, with

275 increased activity in the dmPFC-DMS projection and decreased activity in the dmPFC-BLA

276 projection at avoidance onset. The main findings from our study are summarized in Figure 4L. 


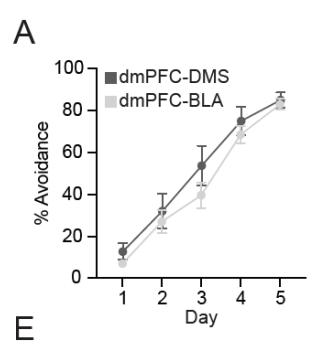

E
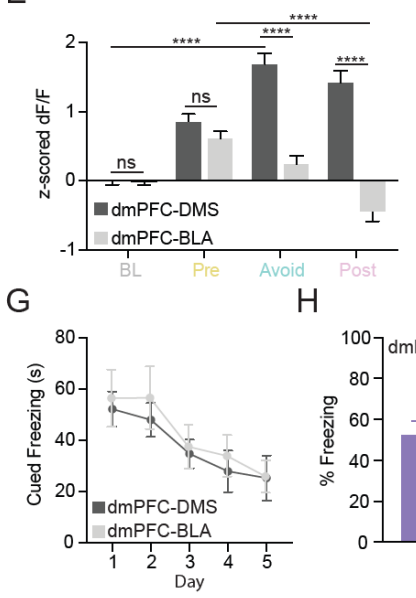

K

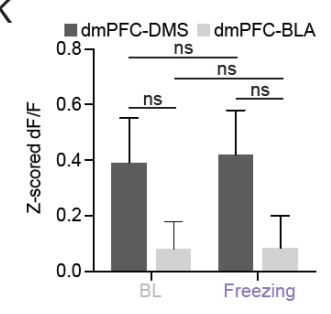

B

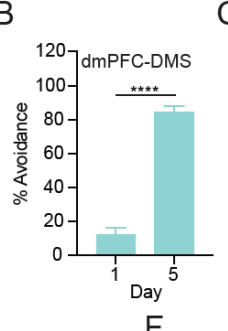

C

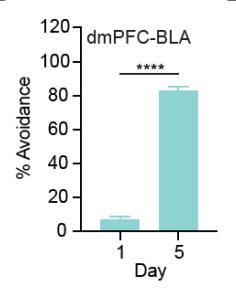

$\mathrm{F}$
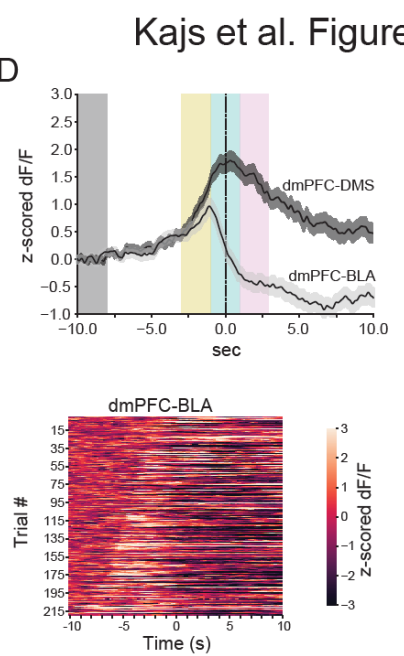

$J$
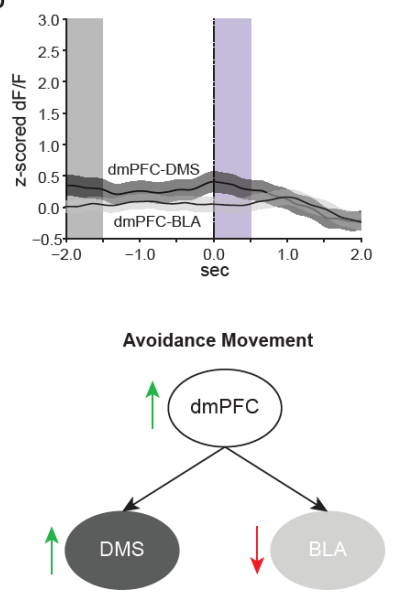

Figure 4. dmPFC-DMS and dmPFC-BLA show divergent encoding of active avoidance behavior. (A) Percent avoidance across training days in the dmPFC-DMS (dark grey line) and dmPFC-BLA (light grey line) cohort. (B-C) Percent avoidance significantly increases from Day 1 to Day 5 in the dmPFC-DMS (left) and dmPFC-BLA (right) cohort. (D) PETH shows increase in calcium signal in the dmPFC-DMS projection and decrease in calcium signal in the dmPFC-BLA projection during avoidance onset. Dark grey line, mean \pm SEM for dmPFC-DMS projection; light grey line, mean \pm SEM for dmPFC-BLA projection; Grey box, baseline period (BL); yellow box, pre avoidance period (Pre); teal box, avoidance period (Avoid); pink box, post avoidance period (Post). (E) Quantification of avoidance PETH shows a significant increase in calcium signal in the avoid (-1 to $1 \mathrm{~s}$ ) period compared to baseline period (-10 to $-8 \mathrm{~s})$ for dmPFC-DMS projection and a significance decrease in signal during the post avoid ( 1 to $3 \mathrm{~s}$ ) period compared to the pre avoid $(-3$ to $-1 \mathrm{~s}$ ) period in the dmPFC-BLA projection. (F) Heatmap of change in calcium signal for individual avoidance trials aligned to avoidance onset and sorted from shortest to longest avoidance latency for the dmPFC-DMS (left) and dmPFC-BLA (right) projections. (G) Percent cued freezing in the dmPFC-DMS (dark grey line) and the dmPFC-BLA (light grey line) cohort. ( $\mathrm{H}-\mathrm{I})$ Percent cued freezing significantly decreases from Day 1 to Day 5 in the dmPFC-DMS (left) and the dmPFC-BLA (right) cohorts. (J) PETH shows no change in calcium signal at freezing onset for either the dmPFC-DMS or the dmPFC-BLA projection. Dark grey line, mean \pm SEM for dmPFC-DMS projection; light grey line, mean \pm SEM for dmPFC-BLA projection; Grey box, baseline period (BL); Purple box, freezing period (Freezing). (K) Quantification of freezing PETH shows no significant change in calcium signal during the freezing period (0-0.5 s) compared to the baseline period (-2 to $-1.5 \mathrm{~s})$. (L) Graphical abstract summarizing main findings from the study. ns = not significant * $p \leq 0.05,{ }^{* *} p \leq 0.01,{ }^{* * * *} p \leq 0.0001$. 


\section{DISCUSSION}

277 We found that the dmPFC and its projections to the DMS and the BLA contain learning-related

278 increases in activity at CS onset during active avoidance. Encoding of active avoidance diverged

279 in the dmPFC-DMS and dmPFC-BLA projections, which showed increased and decreased neural

280 activity at avoidance onset, respectively. To our knowledge, this is the first study to record the

281 endogenous activity of distinct dmPFC projections during active avoidance behavior. Our results

282 reveal the importance of studying projection-defined dmPFC subpopulations as they may play

283 distinct but complementary roles in active avoidance learning and expression.

284 The sharp peak of dmPFC activity at CS onset that significantly increased in amplitude across

285 training suggests that the dmPFC encodes learning-related information for active avoidance

286 behavior. Given that significant differences in neural activity were seen across days but not within

287 days suggests that the learning-related increase in activity at CS onset in the dmPFC is a

288 consolidated phenomenon that gradually builds across time. Another recent study used dmPFC

289 single unit activity to successfully decode CS identity between a CS that predicted shock and led

290 to avoidance (CS+) and a control CS that did not predict shock and did not lead to avoidance

291 (CS-) (Jercog et al., 2021), corroborating our finding that the dmPFC holds active avoidance task-

292 relevant information. While our results show increased dmPFC activity aligned with CS onset,

293 another study in rats using a platform-mediated active avoidance task found inhibition of single

294 dmPFC units upon CS onset unique to avoidance (Diehl et al., 2018). This discrepancy could be

295 explained by the subregion of dmPFC targeted (rostral vs caudal), or technical differences

296 between bulk calcium recording and single unit electrophysiology. For example, calcium

297 indicators are more sensitive to increases rather than decreases in activity and may preferentially

298 reflect synchronous and/or bursting activity of groups of neurons (Chen et al., 2013). Interestingly, 
there was no difference in this CS-evoked neural signal between successful and unsuccessful trials. This observation is supported by other studies (Diehl et al., 2018; Jercog et al., 2021) and suggests that this activity may signal the option to avoid rather than the avoidance behavior itself.

302 Of note, the initial sharp peak of activity upon CS onset was present on the first day before 303 learning had occurred, albeit significantly smaller in amplitude than on the last day of training.

304 Given that the dmPFC receives various sensory related inputs (Ährlund-Richter et al., 2019), this 305 initial peak on day 1 may represent sensory features of the CS, while the increase in amplitude 306 of this peak across days is reflective of learning-related activity. Overall, this is the first study to 307 our knowledge to examine longitudinal learning-related changes in dmPFC activity across days 308 of an active avoidance task.

309 The dmPFC also showed a robust increase in neural activity during avoidance onset in our task.

310 This result is consistent with a recent study employing the platform-mediated avoidance task,

311 which also found increased activity in the dmPFC when animals moved onto a platform to avoid

312 shock. However, there was no difference in the proportion of cells excited between mice trained

313 on fear conditioning or active avoidance in the same apparatus (Diehl et al., 2018), suggesting

314 that increased activity was not specific to avoidance behavior in their task. While their study 315 controlled for locomotion by comparing platform entries between separate avoidance-trained and

316 fear-conditioned cohorts, here we performed a within-animal locomotor control. Comparing 317 dmPFC neural activity during avoidance movements versus intertrial interval movements of 318 similar duration and velocity, we found that the increased neural activity seen during avoidance 319 is not accounted for by general movement alone. This finding is corroborated by another study 320 using dmPFC activity to decode avoidance behavior in a discriminative two-way active avoidance 321 task, which found an increase in decoding accuracy within the last second before the avoidance 322 movement which could not be accounted for by speed (Jercog et al., 2021). The predictive 323 increase in decoder accuracy before avoidance initiation is also in alignment with the increase in 
324 activity we observed in the dmPFC preceding avoidance onset. Furthermore, only the excitatory 325 responses in the dmPFC contained predictive information about avoidance initiation in the 326 discriminative two-way active avoidance task (Jercog et al., 2021), which further supports that 327 notion that the excitatory dmPFC activity we see in our task contains crucial information for proper 328 avoidance performance rather than only encoding movement. We also found differences in 329 dmPFC activity between successful and unsuccessful trials during the period where avoidances 330 normally occur, which has been similarly identified in other studies and may correspond to 331 differences in the behavioral repertoire of the animals during successful and unsuccessful trials 332 (Diehl et al., 2018; Jercog et al., 2021).

333 We propose that the increased dmPFC activity at avoidance onset may be important for the 334 animal to take action in the face of an anxiogenic stimulus. In the active avoidance task, when the 335 CS light is on, dmPFC activity increases when the animal initiates an avoidance movement within 336 the anxiogenic lit chamber of the apparatus. A recent study from our laboratory using the elevated 337 zero maze to assess approach-avoidance conflict showed that dmPFC activity increases as the 338 animal moves into the anxiogenic open arms of the maze (Loewke et al., 2021). These seemingly 339 disparate findings may be reconciled by the idea that dmPFC activity allows the animal to explore 340 or take action in the face of an anxiogenic stimulus, while dmPFC activity decreases once the 341 anxiogenic stimulus has been successfully avoided (i.e., shuttling to the safe chamber in active 342 avoidance, and entering the closed arm of the elevated zero maze). The notion that dmPFC 343 activity may be important for resolving conflicting signals between the drive to explore or take 344 action and the drive to passively cope with an anxiogenic stimulus is supported by various studies 345 suggesting a role for the dmPFC in decision making under conflict (Burgos-Robles et al., 2017; 346 Friedman et al., 2015; Ishikawa et al., 2020; Loewke et al., 2021). 
347 The dmPFC as a whole showed decreased activity during freezing in our active avoidance task,

348 with the duration of this decrease in activity corresponding to the freezing bout length. In contrast,

349 in vivo electrophysiology studies have found increased firing rates in dmPFC neurons during

350 freezing behavior in classical and discriminative fear conditioning tasks (Burgos-Robles et al.,

351 2009; Dejean et al., 2016; Likhtik et al., 2014). Given that calcium indicators are more sensitive

352 to increases rather than decreases in activity (Chen et al., 2013), this difference seems likely

353 unrelated to technique used and may instead be due to key differences in the tasks, such as the

354 fact that the active avoidance task allows for both passive and active coping responses to threat,

355 whereas in classical fear conditioning animals have no control over the shocks and therefore are

356 biased toward passive coping via freezing. Future studies using single cell resolution calcium

357 imaging will help elucidate the encoding of individual dmPFC neurons during freezing in active

358 avoidance versus fear conditioning tasks.

359 Both the dmPFC-DMS and dmPFC-BLA projections showed increased activity at CS onset, with

360 learning-related changes evidenced by significant increases in signal amplitude across training in

361 both projections. As the dmPFC-DMS projection plays an important role in goal-directed behavior

362 (Hart, Bradfield, \& Balleine, 2018; Hart, Bradfield, Fok, et al., 2018), this CS-related activity could

363 hold crucial information regarding action-outcome contingencies for this task. The dmPFC-BLA

364 projection has been linked to associative fear conditioning (Adhikari et al., 2015; Cho et al., 2013)

365 and thus CS-related activity in this projection may contain key information on CS-US associations

366 in this task. When comparing successful and unsuccessful trials, we found no differences in

367 activity during CS onset in either projection, suggesting that CS-related activity in these

368 projections may again signal an avoidance option rather than avoidance behavior itself.

369 Interestingly, downstream BLA neurons do show distinct activity on successful and unsuccessful

370 avoidance trials (Kyriazi et al., 2018). Thus, the BLA likely receives information necessary for

371 distinguishing between these trial types from a region outside the dmPFC. Future studies should 
372 attempt to uncover additional circuits that differentiate between successful and unsuccessful trials

373 that may act upstream of the BLA.

374 While CS-aligned activity looked similar in both projections, they displayed opposing patterns of 375 activity at avoidance onset, with the dmPFC-DMS projection showing increased activity and the 376 dmPFC-BLA projection showing decreased activity. The dmPFC-DMS projection directly 377 interfaces downstream with the striatum which regulates motor control and action selection 378 (Kravitz \& Kreitzer, 2012) and is therefore poised to play a privileged role in aiding avoidance 379 movement initiation. The striatum consists of D1 and D2 medium spiny neurons (MSNs) that, 380 when optogenetically stimulated, drive motor initiation and motor cessation, respectively (Kravitz 381 \& Kreitzer, 2012; Redgrave et al., 2010). The mPFC has stronger synaptic input to D1 versus D2 382 MSNs, and optogenetic stimulation of D1 MSNs recapitulates anxiolytic effects seen with dmPFC383 DMS stimulation (Loewke et al., 2021). Increased activity in the dmPFC-DMS projection may 384 directly excite striatal D1 MSNs leading to motor initiation and, in our task, active avoidance 385 behavior. Conversely, the dmPFC-BLA projection has been tied to freezing behavior, with 386 dmPFC-BLA stimulation during fear conditioning leading to increased freezing at extinction recall 387 (Adhikari et al., 2015). Fear-related information is thought to be sent from the BLA to the central 388 amygdala $(\mathrm{CeA})$ to downstream brainstem structures leading to freezing initiation (Tovote et al., 389 2015). Given that increased activity in the dmPFC-BLA-CeA pathway may promote freezing, the 390 decreased activity we see in the dmPFC-BLA projection during avoidance behavior may help 391 suppress freezing to allow proper active avoidance behavior to occur. The contrasting neural 392 activity in the dmPFC-DMS and dmPFC-BLA projections may therefore play distinct yet 393 complementary roles in coordinating successful active avoidance behavior through the initiation 394 of avoidance movements (dmPFC-DMS) and the suppression of freezing behavior (dmPFC-BLA). 
395 While the dmPFC-DMS projection has not been previously explored within the context of active

396 avoidance, a recent optogenetic study has causally implicated the dmPFC-BLA projection in

397 platform-mediated active avoidance (Diehl et al., 2020). Stimulation of the dmPFC-BLA projection

398 increases avoidance in the platform-mediated task (Diehl et al., 2020), while our photometry

399 results would suggest that inhibiting the dmPFC-BLA projection may increase avoidance given

400 that dmPFC-BLA activity decreases acutely during avoidance in our task. In our previous study

401 examining the dmPFC-DMS and dmPFC-BLA projections during an innate approach-avoidance

402 task, we found that the dmPFC-DMS projection recapitulated whole population dmPFC activity

403 while the dmPFC-BLA projection did not (Loewke et al., 2021). Similarly, here we find that the

404 dmPFC-DMS projection shows increased activity during avoidance similar to the dmPFC overall,

405 while the dmPFC-BLA projection shows distinct decreases in activity during avoidance. The

406 projection-specific activity we observed during avoidance intriguingly parallels fMRI findings

407 during active avoidance in humans (Collins et al., 2014; Delgado et al., 2009). In one study,

408 coupling between the mPFC and the caudate (the human equivalent of the DMS) and between

409 the mPFC and the amygdala during active avoidance trials predicted better active avoidance

410 performance (Collins et al., 2014). The increased coupling between mPFC and caudate/amygdala

411 during active avoidance performance parallels the signals we see in the dmPFC-DMS and

412 dmPFC-BLA projections during active avoidance behavior. The human study also found

413 increased activity in the caudate and decreased activity in the amygdala during active avoidance

414 behavior (Collins et al., 2014), similar to the increased activity in the dmPFC-DMS projection and

415 the decreased activity in the dmPFC-BLA projection we observed during active avoidance.

416 Overall, these results highlight the importance of the mPFC downstream communication with both

417 the dorsal striatum and the amygdala and suggest conservation of function across species in 418 these circuits during active avoidance behavior. 
419 Overall, we find task-relevant information encoding in the dmPFC and its projections to the DMS

420 and the BLA during active avoidance learning, with opposing patterns of activity in the dmPFC-

421 DMS and dmPFC-BLA projections during active avoidance behavior, suggesting that these

422 circuits play distinct but complementary roles in the successful enactment of active avoidance

423 behavior. These findings provide a crucial first step in identifying precise prefrontal subpopulations

424 and circuits for active avoidance behavior that may help guide future treatment targets to alleviate

425 avoidance symptoms seen in anxiety disorders.

\section{MATERIALS AND METHODS}

426

427

428

429

430

431

432

433

434

435

436

437

438

439

440

\section{Animals}

We used wild-type C57BL6/J mice purchased from Jackson Laboratories. Animals were raised in normal light conditions (12:12 light/dark cycle) and given food and water ad libitum. All experiments were conducted in accordance with procedures established by the Institutional Animal Care and Use Committee at the University of California, San Francisco.

\section{Stereotaxic Surgery, Viral Injections, and Fiber Optic Cannula Implantation}

Surgeries were performed at $10-14$ weeks of age. Mice were anesthetized using $5.0 \%$ isoflurane at an oxygen flow rate of $1 \mathrm{~L} / \mathrm{min}$ and placed on top of a heating pad in a stereotaxic apparatus (Kopf Instruments, Tujunga, CA, USA). Anesthesia was maintained with $1.5-2.0 \%$ isoflurane for the duration of the surgery. Respiration and toe pinch response were monitored closely. Slowrelease buprenorphine $(0.5 \mathrm{mg} / \mathrm{kg})$ and ketoprophen $(1.6 \mathrm{mg} / \mathrm{kg})$ were administered subcutaneously at the start of surgery. The incision area was shaved and cleaned with ethanol and betadine. Lidocane $(0.5 \%)$ was administered topically on the scalp. An incision was made along the midline and bregma was measured. Virus was injected (as described below) using a 10 $\mu \mathrm{L}$ nanofil syringe (World Precision Instruments, Sarasota, FL, USA) with a 33-gauge beveled 
441 needle. We used an injection rate of $100 \mathrm{~nL} / \mathrm{min}$ with a 10 -minute delay before retracting the

442 needle. Mice recovered in a clean cage on top of a heating pad and a subsequent injection of

443 ketoprofen $(1.6 \mathrm{mg} / \mathrm{kg})$ was given the following day.

444 For fiber photometry, we injected $500 \mathrm{~nL}$ of AAV5-CaMKII-GCaMP6f or AAV5-CaMKII-eYFP into

445 the dmPFC to record pyramidal neuron activity; to record dmPFC-DMS and dmPFC-BLA 446 projection neurons, we injected $1500 \mathrm{~nL}$ of AAV1-Syn-Flex-GCaMP6m or AAV5-EF1a-DIO-eYFP

447 into the dmPFC and $500 \mathrm{~nL}$ of CAV2-Cre and hSyn-mCherry into the DMS and BLA. Injection

448 coordinates (in millimeters relative to bregma) were as follows: dmPFC (1.8 A/P, $-.35 \mathrm{M} / \mathrm{L},-2.4$

$449 \mathrm{D} / \mathrm{V})$, DMS (.8 A/P, -1.5 M/L, -3.5 D/N), BLA (-1.4 A/P, -3.3 M/L, -4.9 D/V). For all fiber photometry

450 experiments, we implanted a $2.5 \mathrm{~mm}$ metal fiber optic cannula with $400 \mu \mathrm{m}$ fiber optic stub (Doric

451 Lenses, Quebec, Canada) in the dmPFC and waited 4-5 weeks for viral expression. Implant

452 coordinates for the mPFC were $1.8 \mathrm{~A} / \mathrm{P},-.35 \mathrm{M} / \mathrm{L},-2.2 \mathrm{D} / \mathrm{V}$.

453 All viruses were obtained from Addgene, UNC Vector Core, or Institut de Génétique Moléculaire 454 de Montpellier, Montpellier, France.

\section{Active Avoidance Behavior}

456 Mice underwent a two-way active avoidance procedure adapted from Pare 2018. Active 457 avoidance training occurred in a custom made apparatus consisting of two shock floors with strips 458 of visible spectrum LED lights underneath each shock floor. Both shock and light presentations 459 were controlled by an arduino using custom-made arduino code (Arduino, Somerville, MA, USA) 460 in conjunction with location data from video recording software, Ethovision XT (Noldus, 461 Wageningen, Netherlands). All trials were conducted in the dark and infrared lights beneath each 462 shock floor were used to track the animals. Mice underwent 30 active avoidance trials per day for 4635 days. Each active avoidance trial consisted of a 10 second light cue followed by 10 seconds of 
464 light plus $0.3 \mathrm{~mA}$ shock. Light and shock were presented on the shock floor the mouse was

465 currently on at the initiation of the trial. Mice were able to avoid the shock altogether by moving 466 onto the other unlit shock floor during the 10 second light only period. This was considered a 467 successful active avoidance trial. Trials in which the mouse failed to move to the other unlit shock

468 floor during the 10 seconds of light only are considered unsuccessful trials. Training continued 469 until the group average was at or above $80 \%$ successful avoidance (24 out of 30 trials). Location 470 of the mice was recorded and quantified using Ethovision XT software.

\section{$471 \quad$ Fiber Photometry Recording}

472 In vivo calcium data were acquired using a custom-built rig based on a previously described setup 473 (Lerner et al., 2015). This setup was controlled by an RZ5P fiber photometry processor (TDT, 474 Alachua, FL, USA) and Synapse software (TDT). The RZ5P/Synapse software controlled a 4 475 channel LED Driver (DC4100, Thorlabs, Newton, NJ, USA) which in turn controlled two fiber476 coupled LEDS: $470 \mathrm{~nm}$ for GCaMP stimulation and $405 \mathrm{~nm}$ to control for artifactual fluorescence 477 (M470F3, M405FP1, Thorlabs). These LEDs were sinusoidally modulated at $210 \mathrm{~Hz}(470 \mathrm{~nm})$ 478 and $320 \mathrm{~Hz}(405 \mathrm{~nm})$ and connected to a Fluorescence Mini Cube with 4 ports (Doric Lenses) 479 and the combined LEF output was connected through a fiber optic patch cord (0.48 NA, $400 \mu \mathrm{m}$, 480 Doric Lenses) to the cannula via a ceramic sleeve (Thorlabs). The emitted light was focused onto 481 a Visible Femtowatt Photoreceiver Module (Model 2151, Newport, AC low) and sampled at 60 $482 \mathrm{~Hz}$. Video tracking software (Ethovision, Noldus) was synchronized to the photometry setup using 483 TTL pulses generated every 10 seconds following the start of the Noldus trial. Raw photoreceiver 484 data was extracted and analyzed using custom scripts in Matlab (The MathWorks, Natick, MA, 485 USA). The two output signal data was demodulated from the raw signal based on the LED 486 modulation frequency. To normalize the data and correct for bleaching, the $405 \mathrm{~nm}$ channel signal 487 was fitted to a polynomial over time and subtracted from the $470 \mathrm{~nm}$ GCaMP signal, yielding the 488 DF/F value. 


\section{Perfusions and Histology}

490 Following the conclusion of behavioral experiments, animals were anesthetized using $5 \%$

491 isoflurane and given a lethal dose $(1.0 \mathrm{~mL})$ cocktail of ketamine/xylazine $(10 \mathrm{mg} / \mathrm{ml}$ ketamine, 1

$492 \mathrm{mg} / \mathrm{ml}$ xylazine). They were then transcardially perfused with $10 \mathrm{~mL}$ of $1 \mathrm{X}$ PBS followed by $10 \mathrm{~mL}$

$4934 \%$ paraformaldehyde (PFA). Brains were extracted and left in 4\% PFA overnight and then

494 transferred to a $30 \%$ sucrose solution until slicing. The brains were frozen and sliced on a sliding

495 microtome (Leica Biosystems, Wetzlar, Germany) and placed in cryoprotectant in a well-plate.

496 Slices were then washed in 1X PBS, mounted on slides (Fisherbrand Superfrost Plus,

497 ThermoFisher Scientific, Waltham, MA, USA) and air dried (covered). ProLong Gold antifade

498 reagent (Invitrogen, ThermoFisher Scientific) was injected on top of the slices and a cover slip

499 (Slip-rite, ThermoFisher Scientific) was placed on top and the slides were left to dry overnight

500 (covered). Viral injection, fiber photometry cannula implant, and optogenetic cannula implant

501 placements were histologically verified on a fluorescence microscope (Leitz DMRB, Leica).

\section{Movement and Freezing Behavior Analysis}

503 Following the recording of location data using Ethovision, post data collection analysis was 504 performed to identify movement initiations using Ethovision's built in movement detection 505 software. The detection settings used were a 10 sample averaging window, $2.25 \mathrm{~cm} / \mathrm{sec}$ start 506 velocity threshold, and $2 \mathrm{~cm} / \mathrm{sec}$ stop velocity threshold. Additionally, we used open source code 507 (Pennington et al., 2019) to identify freezing. The parameters we used for this analysis were a 508 motion cuttoff of 9.0 , freezing threshold of 1000 , and minimum freeze duration of 25 samples (1 509 second). 
511 Data was analyzed in PyCharm CE (JetBrains, Prague, Czechia) environment. Behavioral,

512 location, and movement initiation data was extracted from both Ethovision and Arduino and

513 synced to Synapse fiber photometry data. From this we extracted the behavioral data (percent

514 avoidance, avoidance latency, and freezing) across all five days of learning. Additionally, we

515 generated peri-event time histograms and heatmaps by time-locking the neural activity (dF/F) and

$516 \quad$ z-scoring the signal to the baseline period (last 10 seconds of inter-trial-interval (ITI) preceding

517 the event). These events included CS (light) onset (also split into successful and unsuccessful

518 trials), avoidance movement initiation (movements during the 10 second light only period of

519 successful trials), and freezing behavior initiation (freezing during the 10 second light only period

520 of all trials). In addition, we also analyzed movement initiations during the ITI periods across all

521 days. The heatmaps for avoidance movements and freezing were sorted by avoidance latency

522 and freezing duration respectively. Quantification was done using the average signal across the

523 following time windows:

524 CS onset: Baseline ( -1 to $0 \mathrm{sec})$, CS response (0 to $1 \mathrm{sec}$ )

525 CS successful vs. unsuccessful: Baseline (-1 to $0 \mathrm{sec}$ ), Initial CS response (0 to $1 \mathrm{sec}$ ), Pre526 avoidance (1 to $2 \mathrm{sec}$ ), Post-avoidance (9 to $10 \mathrm{sec}$ )

527 Avoidance movement: Baseline ( -10 to $-8 \mathrm{sec})$, Pre-avoidance (-3 to $-1 \mathrm{sec})$, Avoidance ( -1 to 1

528 sec), Post-avoid: (1 to $3 \mathrm{sec}$ )

529 ITI movement: Baseline (-10 to $-8 \mathrm{sec})$, Pre-movement $(-3$ to $-1 \mathrm{sec})$, Movement $(-1$ to $1 \mathrm{sec})$,

530 Post-movement: (1 to $3 \mathrm{sec})$

531 Freezing: Baseline (-2 to $-1.5 \mathrm{sec})$, Freezing (0 to $0.5 \mathrm{sec})$

532 All other non-avoidance movement controls were quantified identically to avoidance movement.

533 Lastly, histograms of the distribution of velocity and movement duration for all movement 534 parameters were generated in Prism using a bin width of $1 \mathrm{~cm} / \mathrm{sec}$ and 1 second respectively. 


\section{Statistical Analysis}

536 Statistical Analysis was performed with Prism 8 (Graphpad Software, San Diego, CA, USA).

537 Normality was tested with D'Agostino \& Pearson normality test. Paired t-test (two-tailed, assume

538 gaussian distribution), one-way repeated measures ANOVA with Geisser-Greenhouse correction

539 with Sidak's and Tukey's correction for multiple comparisons, and two-way repeated measures

540 ANOVA with Sidak's and Tukey's correction for multiple comparisons (assume sphericity) was

541 used.

\section{Data and Code Accessibility}

543 All data and code are freely available through contacting the corresponding author directly.

\section{ACKNOWLEDGEMENTS}

545 We would like to acknowledge Dr. Pinelopi Kyriazi and Dr. Drew B. Headley from Dr. Denis Pare's

546 lab for their guidance regarding construction of the active avoidance apparatus and for sharing 547 custom arduino code for running the active avoidance experiments.

\section{$548 \quad$ FUNDING}

549 LAG is funded by a Chan Zuckerberg Biohub award and a Kavli Institute for Fundamental 550 Neuroscience award.

\section{COMPETING INTERESTS}

552 All authors declare no competing interests. 


\section{REFERENCES}

Adhikari, A., Lerner, T. N., Finkelstein, J., Pak, S., Jennings, J. H., Davidson, T. J., Ferenczi, E., Gunaydin, L. A., Mirzabekov, J. J., Ye, L., Kim, S.-Y., Lei, A., \& Deisseroth, K. (2015). Basomedial amygdala mediates top-down control of anxiety and fear. Nature, 527(7577), 179-185. https://doi.org/10.1038/nature15698

Ährlund-Richter, S., Xuan, Y., van Lunteren, J. A., Kim, H., Ortiz, C., Pollak Dorocic, I., Meletis, K., \& Carlén, M. (2019). A whole-brain atlas of monosynaptic input targeting four different cell types in the medial prefrontal cortex of the mouse. Nature Neuroscience, 22(4), 657668. https://doi.org/10.1038/s41593-019-0354-y

Amorapanth, P., LeDoux, J. E., \& Nader, K. (2000). Different lateral amygdala outputs mediate reactions and actions elicited by a fear-arousing stimulus. Nature Neuroscience, 3(1), 7479. https://doi.org/10.1038/71145

Balleine, B. W., \& O'Doherty, J. P. (2010). Human and rodent homologies in action control: corticostriatal determinants of goal-directed and habitual action.

Neuropsychopharmacology: Official Publication of the American College of Neuropsychopharmacology, 35(1), 48-69. https://doi.org/10.1038/npp.2009.131

Beck, K. D., Jiao, X., Smith, I. M., Myers, C. E., Pang, K. C. H., \& Servatius, R. J. (2014). ITISignals and Prelimbic Cortex Facilitate Avoidance Acquisition and Reduce Avoidance Latencies, Respectively, in Male WKY Rats. Frontiers in Behavioral Neuroscience, 8, 403. https://doi.org/10.3389/fnbeh.2014.00403

Boeke, E. A., Moscarello, J. M., LeDoux, J. E., Phelps, E. A., \& Hartley, C. A. (2017). Active Avoidance: Neural Mechanisms and Attenuation of Pavlovian Conditioned Responding. The Journal of Neuroscience: The Official Journal of the Society for Neuroscience, 37(18), 4808-4818. https://doi.org/10.1523/JNEUROSCI.3261-16.2017

Boschen, S. L., Wietzikoski, E. C., Winn, P., \& Da Cunha, C. (2011). The role of nucleus 
accumbens and dorsolateral striatal D2 receptors in active avoidance conditioning.

Neurobiology of Learning and Memory, 96(2), 254-262.

https://doi.org/10.1016/j.nlm.2011.05.002

Bravo-Rivera, C., Roman-Ortiz, C., Brignoni-Perez, E., Sotres-Bayon, F., \& Quirk, G. J. (2014).

Neural structures mediating expression and extinction of platform-mediated avoidance. The Journal of Neuroscience: The Official Journal of the Society for Neuroscience, 34(29), 9736-9742. https://doi.org/10.1523/JNEUROSCI.0191-14.2014

Bravo-Rivera, C., Roman-Ortiz, C., Montesinos-Cartagena, M., \& Quirk, G. J. (2015). Persistent active avoidance correlates with activity in prelimbic cortex and ventral striatum. Frontiers in Behavioral Neuroscience, 9, 184. https://doi.org/10.3389/fnbeh.2015.00184

Burgos-Robles, A., Kimchi, E. Y., Izadmehr, E. M., Porzenheim, M. J., Ramos-Guasp, W. A., Nieh, E. H., Felix-Ortiz, A. C., Namburi, P., Leppla, C. A., Presbrey, K. N., Anandalingam, K. K., Pagan-Rivera, P. A., Anahtar, M., Beyeler, A., \& Tye, K. M. (2017). Amygdala inputs to prefrontal cortex guide behavior amid conflicting cues of reward and punishment. Nature Neuroscience, 20(6), 824-835. https://doi.org/10.1038/nn.4553

Burgos-Robles, A., Vidal-Gonzalez, I., \& Quirk, G. J. (2009). Sustained conditioned responses in prelimbic prefrontal neurons are correlated with fear expression and extinction failure. The Journal of Neuroscience: The Official Journal of the Society for Neuroscience, 29(26), 8474-8482. https://doi.org/10.1523/JNEUROSCI.0378-09.2009

Capuzzo, G., \& Floresco, S. B. (2020). Prelimbic and Infralimbic Prefrontal Regulation of Active and Inhibitory Avoidance and Reward-Seeking. The Journal of Neuroscience: The Official Journal of the Society for Neuroscience, 40(24), 4773-4787.

https://doi.org/10.1523/JNEUROSCI.0414-20.2020

Chen, T.-W., Wardill, T. J., Sun, Y., Pulver, S. R., Renninger, S. L., Baohan, A., Schreiter, E. R., Kerr, R. A., Orger, M. B., Jayaraman, V., Looger, L. L., Svoboda, K., \& Kim, D. S. (2013). Ultra-sensitive fluorescent proteins for imaging neuronal activity. Published as: Nature, 
499(7458), 295-300.

Choi, J.-S., Cain, C. K., \& LeDoux, J. E. (2010). The role of amygdala nuclei in the expression of auditory signaled two-way active avoidance in rats. Learning \& Memory , 17(3), 139-147. https://doi.org/10.1101//m.1676610

Cho, J.-H., Deisseroth, K., \& Bolshakov, V. Y. (2013). Synaptic encoding of fear extinction in mPFC-amygdala circuits. Neuron, 80(6), 1491-1507.

https://doi.org/10.1016/j.neuron.2013.09.025

Collins, K. A., Mendelsohn, A., Cain, C. K., \& Schiller, D. (2014). Taking action in the face of threat: neural synchronization predicts adaptive coping. The Journal of Neuroscience: The Official Journal of the Society for Neuroscience, 34(44), 14733-14738.

https://doi.org/10.1523/JNEUROSCI.2152-14.2014

Corcoran, K. A., \& Quirk, G. J. (2007). Activity in prelimbic cortex is necessary for the expression of learned, but not innate, fears. The Journal of Neuroscience: The Official Journal of the Society for Neuroscience, 27(4), 840-844.

https://doi.org/10.1523/JNEUROSCI.5327-06.2007

Courtin, J., Chaudun, F., Rozeske, R. R., Karalis, N., Gonzalez-Campo, C., Wurtz, H., Abdi, A., Baufreton, J., Bienvenu, T. C. M., \& Herry, C. (2014). Prefrontal parvalbumin interneurons shape neuronal activity to drive fear expression. Nature, 505(7481), 92-96. https://doi.org/10.1038/nature12755

Darvas, M., Fadok, J. P., \& Palmiter, R. D. (2011). Requirement of dopamine signaling in the amygdala and striatum for learning and maintenance of a conditioned avoidance response. Learning \& Memory , 18(3), 136-143. https://doi.org/10.1101//m.2041211

Dejean, C., Courtin, J., Karalis, N., Chaudun, F., Wurtz, H., Bienvenu, T. C. M., \& Herry, C. (2016). Prefrontal neuronal assemblies temporally control fear behaviour. Nature, 535(7612), 420-424. https://doi.org/10.1038/nature18630

Delgado, M. R., Jou, R. L., Ledoux, J. E., \& Phelps, E. A. (2009). Avoiding negative outcomes: 
tracking the mechanisms of avoidance learning in humans during fear conditioning.

Frontiers in Behavioral Neuroscience, 3, 33. https://doi.org/10.3389/neuro.08.033.2009

Diehl, M. M., Bravo-Rivera, C., Rodriguez-Romaguera, J., Pagan-Rivera, P. A., Burgos-Robles, A., Roman-Ortiz, C., \& Quirk, G. J. (2018). Active avoidance requires inhibitory signaling in the rodent prelimbic prefrontal cortex. eLife, 7. https://doi.org/10.7554/eLife.34657

Diehl, M. M., Iravedra-Garcia, J. M., Morán-Sierra, J., Rojas-Bowe, G., Gonzalez-Diaz, F. N., Valentín-Valentín, V. P., \& Quirk, G. J. (2020). Divergent projections of the prelimbic cortex bidirectionally regulate active avoidance. eLife, 9. https://doi.org/10.7554/eLife.59281

Dombrowski, P. A., Maia, T. V., Boschen, S. L., Bortolanza, M., Wendler, E., Schwarting, R. K. W., Brandão, M. L., Winn, P., Blaha, C. D., \& Da Cunha, C. (2013). Evidence that conditioned avoidance responses are reinforced by positive prediction errors signaled by tonic striatal dopamine. Behavioural Brain Research, 241, 112-119. https://doi.org/10.1016/j.bbr.2012.06.031

Duvarci, S., \& Pare, D. (2014). Amygdala microcircuits controlling learned fear. Neuron, 82(5), 966-980. https://doi.org/10.1016/j.neuron.2014.04.042

Fenton, G. E., Pollard, A. K., Halliday, D. M., Mason, R., Bredy, T. W., \& Stevenson, C. W. (2014). Persistent prelimbic cortex activity contributes to enhanced learned fear expression in females. Learning \& Memory , 21(2), 55-60. https://doi.org/10.1101//m.033514.113 Friedman, A., Homma, D., Gibb, L. G., Amemori, K.-I., Rubin, S. J., Hood, A. S., Riad, M. H., \& Graybiel, A. M. (2015). A Corticostriatal Path Targeting Striosomes Controls DecisionMaking under Conflict. Cell, 161(6), 1320-1333. https://doi.org/10.1016/j.cell.2015.04.049 Garrido, P., De Blas, M., Giné, E., Santos, Á., \& Mora, F. (2012). Aging impairs the control of prefrontal cortex on the release of corticosterone in response to stress and on memory consolidation. Neurobiology of Aging, 33(4), 827.e1-e9. https://doi.org/10.1016/j.neurobiolaging.2011.06.011

Gentry, R. N., Lee, B., \& Roesch, M. R. (2016). Phasic dopamine release in the rat nucleus 
accumbens predicts approach and avoidance performance. Nature Communications, 7, 13154. https://doi.org/10.1038/ncomms13154

Giustino, T. F., \& Maren, S. (2015). The Role of the Medial Prefrontal Cortex in the Conditioning and Extinction of Fear. Frontiers in Behavioral Neuroscience, 9, 298.

https://doi.org/10.3389/fnbeh.2015.00298

Gourley, S. L., \& Taylor, J. R. (2016). Going and stopping: Dichotomies in behavioral control by the prefrontal cortex. Nature Neuroscience, 19(6), 656-664.

https://doi.org/10.1038/nn.4275

Grace, A. A., Floresco, S. B., Goto, Y., \& Lodge, D. J. (2007). Regulation of firing of dopaminergic neurons and control of goal-directed behaviors. Trends in Neurosciences, 30(5), 220-227. https://doi.org/10.1016/j.tins.2007.03.003

Gremel, C. M., \& Costa, R. M. (2013). Orbitofrontal and striatal circuits dynamically encode the shift between goal-directed and habitual actions. Nature Communications, 4, 2264. https://doi.org/10.1038/ncomms3264

Hart, G., Bradfield, L. A., \& Balleine, B. W. (2018). Prefrontal Corticostriatal Disconnection Blocks the Acquisition of Goal-Directed Action. The Journal of Neuroscience: The Official Journal of the Society for Neuroscience, 38(5), 1311-1322.

https://doi.org/10.1523/JNEUROSCI.2850-17.2017

Hart, G., Bradfield, L. A., Fok, S. Y., Chieng, B., \& Balleine, B. W. (2018). The Bilateral Prefronto-striatal Pathway Is Necessary for Learning New Goal-Directed Actions. Current Biology: CB, 28(14), 2218-2229.e7. https://doi.org/10.1016/j.cub.2018.05.028

Herry, C., \& Johansen, J. P. (2014). Encoding of fear learning and memory in distributed neuronal circuits. Nature Neuroscience, 17(12), 1644-1654. https://doi.org/10.1038/nn.3869

Holzschneider, K., \& Mulert, C. (2011). Neuroimaging in anxiety disorders. Dialogues in Clinical Neuroscience, 13(4), 453-461. https://www.ncbi.nlm.nih.gov/pubmed/22275850 
Huang, W.-C., Zucca, A., Levy, J., \& Page, D. T. (2020). Social Behavior Is Modulated by Valence-Encoding mPFC-Amygdala Sub-circuitry. Cell Reports, 32(2), 107899. https://doi.org/10.1016/j.celrep.2020.107899

Ishikawa, J., Sakurai, Y., Ishikawa, A., \& Mitsushima, D. (2020). Contribution of the prefrontal cortex and basolateral amygdala to behavioral decision-making under reward/punishment conflict. Psychopharmacology, 237(3), 639-654. https://doi.org/10.1007/s00213-01905398-7

Ito, W., \& Morozov, A. (2019). Prefrontal-amygdala plasticity enabled by observational fear. Neuropsychopharmacology: Official Publication of the American College of Neuropsychopharmacology, 44(10), 1778-1787. https://doi.org/10.1038/s41386-019-03427

Izquierdo, L. A., Barros, D. M., da Costa, J. C., Furini, C., Zinn, C., Cammarota, M., Bevilaqua, L. R., \& Izquierdo, I. (2007). A link between role of two prefrontal areas in immediate memory and in long-term memory consolidation. Neurobiology of Learning and Memory, 88(2), 160-166. https://doi.org/10.1016/j.nIm.2007.04.014

Jercog, D., Winke, N., Sung, K., Fernandez, M. M., Francioni, C., Rajot, D., Courtin, J., Chaudun, F., Jercog, P. E., Valerio, S., \& Herry, C. (2021). Dynamical prefrontal population coding during defensive behaviours. Nature, 595(7869), 690-694.

https://doi.org/10.1038/s41586-021-03726-6

Killcross, S., Robbins, T. W., \& Everitt, B. J. (1997). Different types of fear-conditioned behaviour mediated by separate nuclei within amygdala. Nature, 388(6640), 377-380. https://doi.org/10.1038/41097

Klavir, O., Prigge, M., Sarel, A., Paz, R., \& Yizhar, O. (2017). Manipulating fear associations via optogenetic modulation of amygdala inputs to prefrontal cortex. Nature Neuroscience, 20(6), 836-844. https://doi.org/10.1038/nn.4523

Kravitz, A. V., \& Kreitzer, A. C. (2012). Striatal mechanisms underlying movement, 
reinforcement, and punishment. Physiology , 27(3), 167-177.

https://doi.org/10.1152/physiol.00004.2012

Kyriazi, P., Headley, D. B., \& Pare, D. (2018). Multi-dimensional Coding by Basolateral Amygdala Neurons. Neuron, 99(6), 1315-1328.e5.

https://doi.org/10.1016/j.neuron.2018.07.036

Lázaro-Muñoz, G., LeDoux, J. E., \& Cain, C. K. (2010). Sidman instrumental avoidance initially depends on lateral and basal amygdala and is constrained by central amygdala-mediated Pavlovian processes. Biological Psychiatry, 67(12), 1120-1127.

https://doi.org/10.1016/j.biopsych.2009.12.002

LeDoux, J. E., Moscarello, J., Sears, R., \& Campese, V. (2017). The birth, death and resurrection of avoidance: a reconceptualization of a troubled paradigm. Molecular Psychiatry, 22(1), 24-36. https://doi.org/10.1038/mp.2016.166

Lee, A. T., Vogt, D., Rubenstein, J. L., \& Sohal, V. S. (2014). A class of GABAergic neurons in the prefrontal cortex sends long-range projections to the nucleus accumbens and elicits acute avoidance behavior. The Journal of Neuroscience: The Official Journal of the Society for Neuroscience, 34(35), 11519-11525. https://doi.org/10.1523/JNEUROSCI.115714.2014

Likhtik, E., Stujenske, J. M., Topiwala, M. A., Harris, A. Z., \& Gordon, J. A. (2014). Prefrontal entrainment of amygdala activity signals safety in learned fear and innate anxiety. Nature Neuroscience, 17(1), 106-113. https://doi.org/10.1038/nn.3582

Loewke, A. C., Minerva, A. R., Nelson, A. B., Kreitzer, A. C., \& Gunaydin, L. A. (2021). Frontostriatal Projections Regulate Innate Avoidance Behavior. The Journal of Neuroscience: The Official Journal of the Society for Neuroscience, 41(25), 5487-5501. https://doi.org/10.1523/JNEUROSCI.2581-20.2021

Marek, R., Xu, L., Sullivan, R. K. P., \& Sah, P. (2018). Excitatory connections between the prelimbic and infralimbic medial prefrontal cortex show a role for the prelimbic cortex in fear 
extinction. Nature Neuroscience, 21(5), 654-658. https://doi.org/10.1038/s41593-018-0137X

Maren, S., Poremba, A., \& Gabriel, M. (1991). Basolateral amygdaloid multi-unit neuronal correlates of discriminative avoidance learning in rabbits. Brain Research, 549(2), 311-316. https://doi.org/10.1016/0006-8993(91)90473-9

Meyer, H. C., Odriozola, P., Cohodes, E. M., Mandell, J. D., Li, A., Yang, R., Hall, B. S., Haberman, J. T., Zacharek, S. J., Liston, C., Lee, F. S., \& Gee, D. G. (2019). Ventral hippocampus interacts with prelimbic cortex during inhibition of threat response via learned safety in both mice and humans. Proceedings of the National Academy of Sciences of the United States of America. https://doi.org/10.1073/pnas.1910481116

Oleson, E. B., Gentry, R. N., Chioma, V. C., \& Cheer, J. F. (2012). Subsecond dopamine release in the nucleus accumbens predicts conditioned punishment and its successful avoidance. The Journal of Neuroscience: The Official Journal of the Society for Neuroscience, 32(42), 14804-14808. https://doi.org/10.1523/JNEUROSCI.3087-12.2012 Pennington, Z. T., Dong, Z., Feng, Y., Vetere, L. M., Page-Harley, L., Shuman, T., \& Cai, D. J. (2019). ezTrack: An open-source video analysis pipeline for the investigation of animal behavior. Scientific Reports, 9(1), 19979. https://doi.org/10.1038/s41598-019-56408-9

Peters, Y. M., O’Donnell, P., \& Carelli, R. M. (2005). Prefrontal cortical cell firing during maintenance, extinction, and reinstatement of goal-directed behavior for natural reward. Synapse , 56(2), 74-83. https://doi.org/10.1002/syn.20129

Piantadosi, P. T., Yeates, D. C. M., \& Floresco, S. B. (2018). Cooperative and dissociable involvement of the nucleus accumbens core and shell in the promotion and inhibition of actions during active and inhibitory avoidance. Neuropharmacology, 138, 57-71. https://doi.org/10.1016/j.neuropharm.2018.05.028

Pinto, L., \& Dan, Y. (2015). Cell-Type-Specific Activity in Prefrontal Cortex during Goal-Directed Behavior. Neuron, 87(2), 437-450. https://doi.org/10.1016/j.neuron.2015.06.021 
Pitts, E. G., Li, D. C., \& Gourley, S. L. (2018). Bidirectional coordination of actions and habits by TrkB in mice. Scientific Reports, 8(1), 4495. https://doi.org/10.1038/s41598-018-22560-x

Poremba, A., \& Gabriel, M. (1999). Amygdala neurons mediate acquisition but not maintenance of instrumental avoidance behavior in rabbits. The Journal of Neuroscience: The Official Journal of the Society for Neuroscience, 19(21), 9635-9641.

https://www.ncbi.nlm.nih.gov/pubmed/10531465

Ramirez, F., Moscarello, J. M., LeDoux, J. E., \& Sears, R. M. (2015). Active avoidance requires a serial basal amygdala to nucleus accumbens shell circuit. The Journal of Neuroscience: The Official Journal of the Society for Neuroscience, 35(8), 3470-3477. https://doi.org/10.1523/JNEUROSCI.1331-14.2015

Rauch, S. L., \& Shin, L. M. (2002). Structural and functional imaging of anxiety and stress disorders. In Neuropsychopharmacology: The Fifth Generation of Progress (pp. 953-966).

Redgrave, P., Rodriguez, M., Smith, Y., Rodriguez-Oroz, M. C., Lehericy, S., Bergman, H., Agid, Y., DeLong, M. R., \& Obeso, J. A. (2010). Goal-directed and habitual control in the basal ganglia: implications for Parkinson's disease. Nature Reviews. Neuroscience, 11(11), 760-772. https://doi.org/10.1038/nrn2915

Rodriguez-Romaguera, J., Greenberg, B. D., Rasmussen, S. A., \& Quirk, G. J. (2016). An Avoidance-Based Rodent Model of Exposure With Response Prevention Therapy for Obsessive-Compulsive Disorder. Biological Psychiatry, 80(7), 534-540. https://doi.org/10.1016/j.biopsych.2016.02.012

Sharpe, M. J., \& Killcross, S. (2014). The prelimbic cortex uses higher-order cues to modulate both the acquisition and expression of conditioned fear. Frontiers in Systems Neuroscience, 8, 235. https://doi.org/10.3389/fnsys.2014.00235

Sierra-Mercado, D., Padilla-Coreano, N., \& Quirk, G. J. (2011). Dissociable roles of prelimbic and infralimbic cortices, ventral hippocampus, and basolateral amygdala in the expression and extinction of conditioned fear. Neuropsychopharmacology: Official Publication of the 
American College of Neuropsychopharmacology, 36(2), 529-538.

https://doi.org/10.1038/npp.2010.184

Sotres-Bayon, F., Sierra-Mercado, D., Pardilla-Delgado, E., \& Quirk, G. J. (2012). Gating of fear in prelimbic cortex by hippocampal and amygdala inputs. Neuron, 76(4), 804-812.

https://doi.org/10.1016/j.neuron.2012.09.028

Sripada, R. K., Garfinkel, S. N., \& Liberzon, I. (2013). Avoidant symptoms in PTSD predict fear circuit activation during multimodal fear extinction. Frontiers in Human Neuroscience, 7, 672. https://doi.org/10.3389/fnhum.2013.00672

Stelly, C. E., Haug, G. C., Fonzi, K. M., Garcia, M. A., Tritley, S. C., Magnon, A. P., Ramos, M. A. P., \& Wanat, M. J. (2019). Pattern of dopamine signaling during aversive events predicts active avoidance learning. Proceedings of the National Academy of Sciences of the United States of America, 116(27), 13641-13650. https://doi.org/10.1073/pnas.1904249116

Terburg, D., Scheggia, D., Triana Del Rio, R., Klumpers, F., Ciobanu, A. C., Morgan, B., Montoya, E. R., Bos, P. A., Giobellina, G., van den Burg, E. H., de Gelder, B., Stein, D. J., Stoop, R., \& van Honk, J. (2018). The Basolateral Amygdala Is Essential for Rapid Escape: A Human and Rodent Study. Cell, 175(3), 723-735.e16.

https://doi.org/10.1016/j.cell.2018.09.028

Torres-García, M. E., Medina, A. C., Quirarte, G. L., \& Prado-Alcalá, R. A. (2017). Differential Effects of Inactivation of Discrete Regions of Medial Prefrontal Cortex on Memory Consolidation of Moderate and Intense Inhibitory Avoidance Training. Frontiers in Pharmacology, 8, 842. https://doi.org/10.3389/fphar.2017.00842

Tovote, P., Fadok, J. P., \& Lüthi, A. (2015). Neuronal circuits for fear and anxiety. Nature Reviews. Neuroscience, 16(6), 317-331. https://doi.org/10.1038/nrn3945

Vander Weele, C. M., Siciliano, C. A., Matthews, G. A., Namburi, P., Izadmehr, E. M., Espinel, I. C., Nieh, E. H., Schut, E. H. S., Padilla-Coreano, N., Burgos-Robles, A., Chang, C.-J., Kimchi, E. Y., Beyeler, A., Wichmann, R., Wildes, C. P., \& Tye, K. M. (2018). Dopamine 
enhances signal-to-noise ratio in cortical-brainstem encoding of aversive stimuli. Nature, 563(7731), 397-401. https://doi.org/10.1038/s41586-018-0682-1

Wendler, E., Gaspar, J. C. C., Ferreira, T. L., Barbiero, J. K., Andreatini, R., Vital, M. A. B. F., Blaha, C. D., Winn, P., \& Da Cunha, C. (2014). The roles of the nucleus accumbens core, dorsomedial striatum, and dorsolateral striatum in learning: performance and extinction of Pavlovian fear-conditioned responses and instrumental avoidance responses. Neurobiology of Learning and Memory, 109, 27-36. https://doi.org/10.1016/j.nlm.2013.11.009

Wenzel, J. M., Oleson, E. B., Gove, W. N., Cole, A. B., Gyawali, U., Dantrassy, H. M., Bluett, R. J., Dryanovski, D. I., Stuber, G. D., Deisseroth, K., Mathur, B. N., Patel, S., Lupica, C. R., \& Cheer, J. F. (2018). Phasic Dopamine Signals in the Nucleus Accumbens that Cause Active Avoidance Require Endocannabinoid Mobilization in the Midbrain. Current Biology: CB, 28(9), 1392-1404.e5. https://doi.org/10.1016/j.cub.2018.03.037

Wietzikoski, E. C., Boschen, S. L., Miyoshi, E., Bortolanza, M., Dos Santos, L. M., Frank, M., Brandão, M. L., Winn, P., \& Da Cunha, C. (2012). Roles of D1-like dopamine receptors in the nucleus accumbens and dorsolateral striatum in conditioned avoidance responses. Psychopharmacology, 219(1), 159-169. https://doi.org/10.1007/s00213-011-2384-3 Yin, H. H., \& Knowlton, B. J. (2006). The role of the basal ganglia in habit formation. Nature Reviews. Neuroscience, 7(6), 464-476. https://doi.org/10.1038/nrn1919 Zhang, Y., Fukushima, H., \& Kida, S. (2011). Induction and requirement of gene expression in the anterior cingulate cortex and medial prefrontal cortex for the consolidation of inhibitory avoidance memory. Molecular Brain, 4, 4. https://doi.org/10.1186/1756-6606-4-4 


\section{SUPPLEMENTAL FIGURES}

A dmPFC Photometry GCaMP

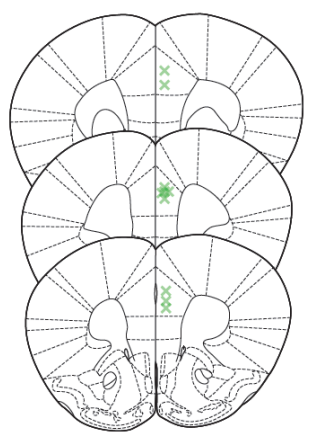

Bregma 1.94 to 1.70 GCaMP $\times$
B

\section{Kajs et al. Supplemental Figure 1}

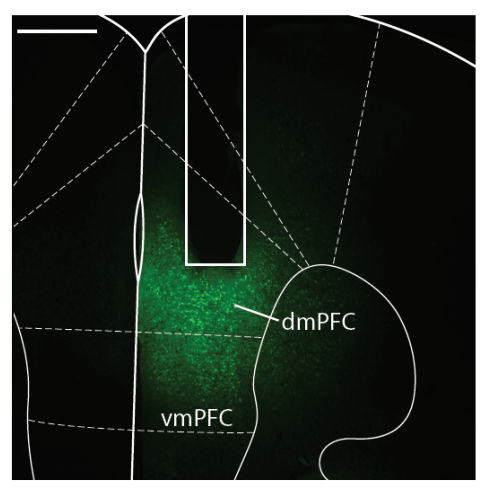

Supplemental Figure 1. Histology and targeting for dmPFC photometry surgeries. (A) Verification of GCaMP virus injection in dmPFC ( $N=10$ mice). (B) Representative histological image of fiber photometry implant and GCAMP viral expression in dmPFC. Scale bar $500 \mu \mathrm{m}$. 


\section{Kajs et al. Supplemental Figure 2}

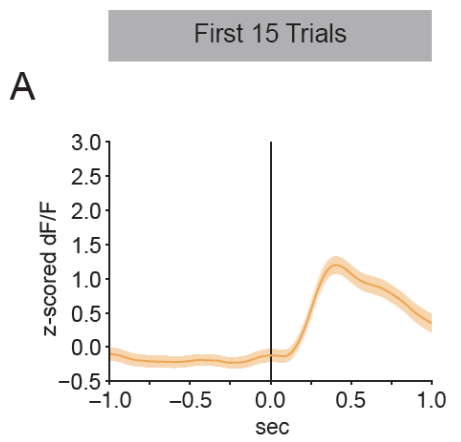

C
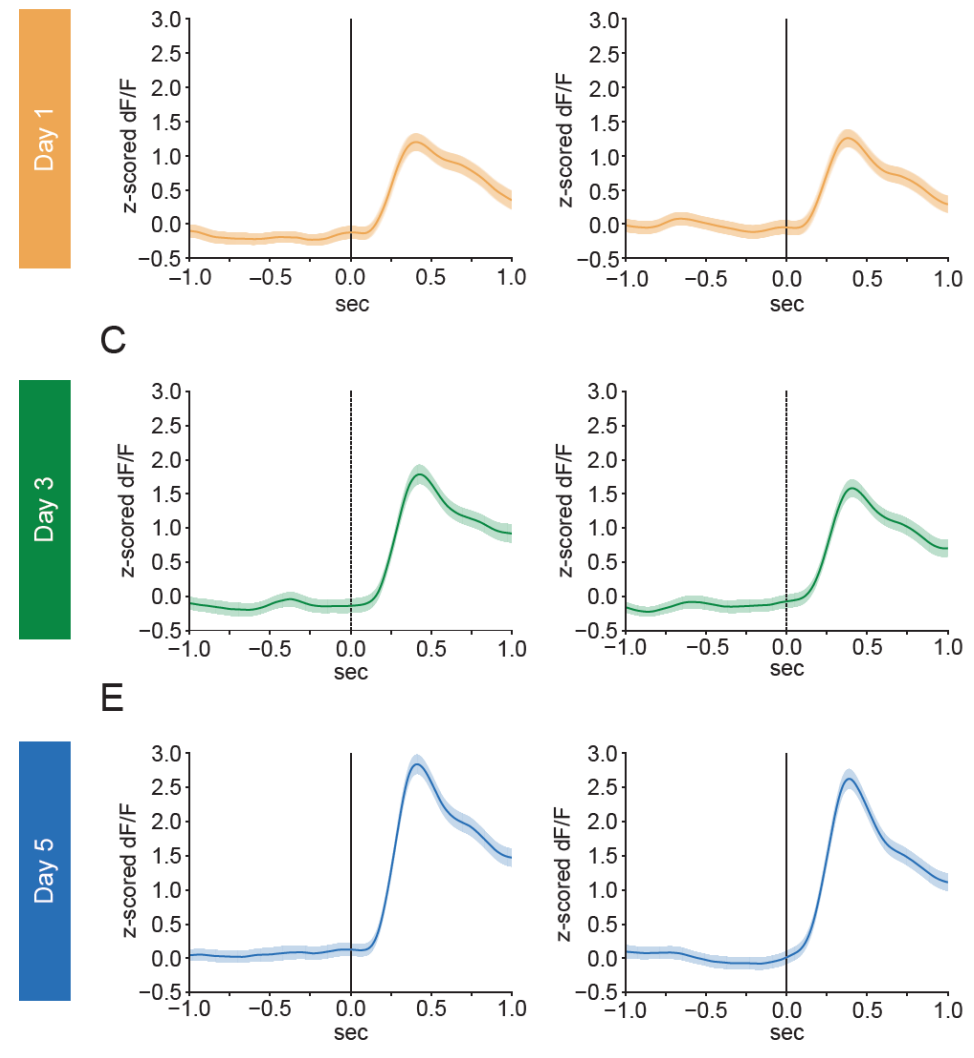

B
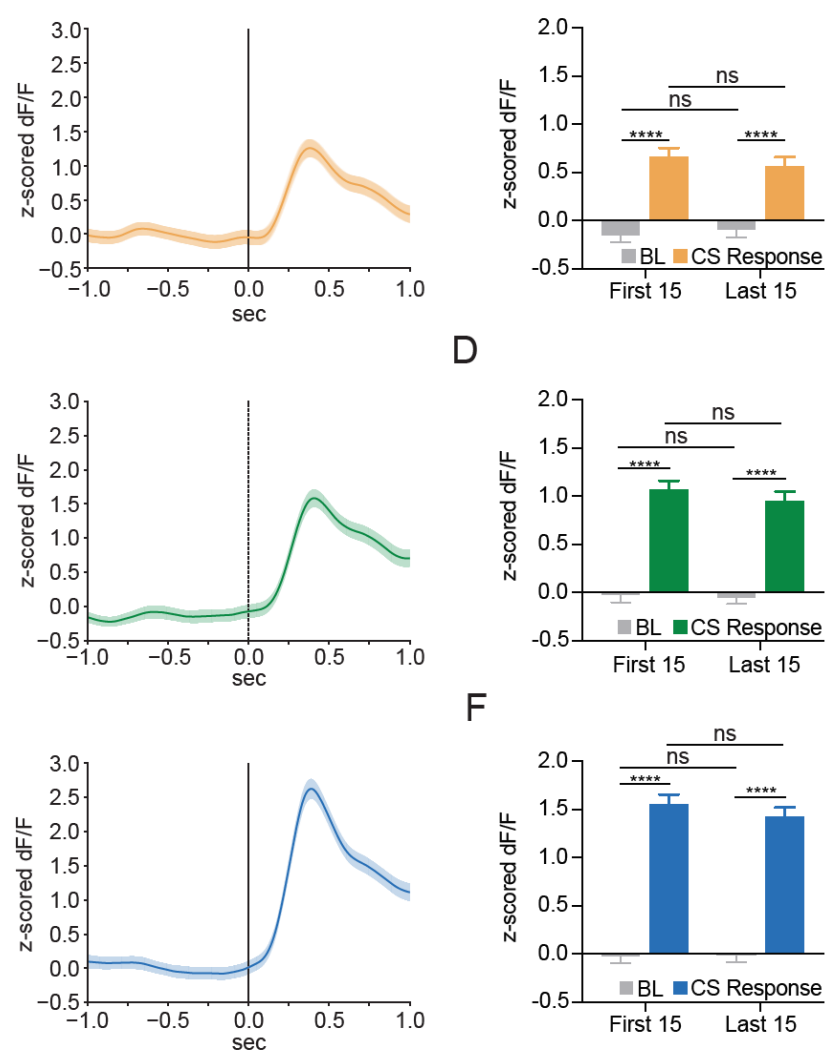

F

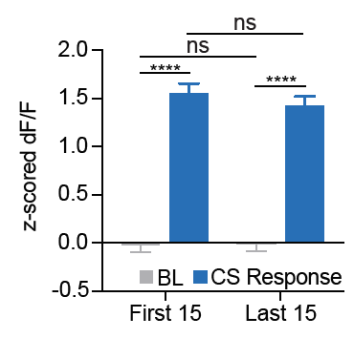

Supplemental Figure 2. No within-day differences in dmPFC neural activity at CS onset. (A) PETHs of dmPFC calcium signal show no differences between the first 15 trials (left) and the last 15 trials (right) on day 1 of training. (B) Quantification of the day 1 PETHs show no significant differences in calcium signal between the first 15 trials and the last 15 trials during the baseline (-1 to $0 \mathrm{~s}$ ) or CS (0 to $1 \mathrm{~s})$ periods (Twoway ANOVA, Part of Session $x$ Task Period $p=0.3176$, Part of Session $p=0.8385$, Task Period $p<0.0001$; Sidak's Multiple Comparisons Test, First 15 Baseline vs Last 15 Baseline $p=0.994$, First 15 Baseline vs First 15 CS $p<0.0001$, First 15 CS vs Last 15 CS $p=0.9509$, Last 15 Baseline vs Last 15 CS $p<0.0001$; $N=10$ mice, First $15 n=150$ trials, Last $15 n=150$ trials). (C) PETHs of dmPFC calcium signal show no differences between the first 15 trials (left) and the last 15 trials (right) on day 3 of training. (D) Quantification of the day 3 PETHs show no significant differences in calcium signal between the first 15 trials and the last 15 trials during the baseline (-1 to $0 \mathrm{~s}$ ) or CS (0 to $1 \mathrm{~s}$ ) periods (Two-way ANOVA, Part of Session $x$ Task Period $p=0.6153$, Part of Session $p=0.3854$, Task Period $p<0.0001$; Sidak's Multiple Comparisons Test, First 15 Baseline vs Last 15 Baseline $p>0.9999$, First 15 Baseline vs First 15 CS $p<0.0001$, First 15 CS vs Last 15 CS $p=0.9116$, Last 15 Baseline vs Last 15 CS $p<0.0001 ; N=10$ mice, First $15 n=150$ trials, Last $15 n=150$ trials). (E) PETHs of dmPFC calcium signal show no differences between the first 15 trials (left) and the last 15 trials (right) on day 5 of training. (F) Quantification of the day 5 PETHs show no significant differences in calcium signal between the first 15 trials and the last 15 trials during the baseline $(-1$ to $0 \mathrm{~s}$ ) or CS (0 to $1 \mathrm{~s}$ ) periods (Two-way ANOVA, Part of Session $x$ Task Period $p=0.388$, Part of Session $p=0.4610$, Task Period $p<0.0001$; Sidak's Multiple Comparisons Test, First 15 Baseline vs Last 15 Baseline $p>0.9999$, First 15 Baseline vs First 15 CS $p<0.0001$, First 15 CS vs Last 15 CS $p=0.8329$, Last 15 Baseline vs Last 15 CS $p<0.0001 ; N=10$ mice, First $15 n=150$ trials, Last $15 n=150$ trials). ns $=$ not significant, ${ }^{* * * *} p \leq 0.0001$. 
A

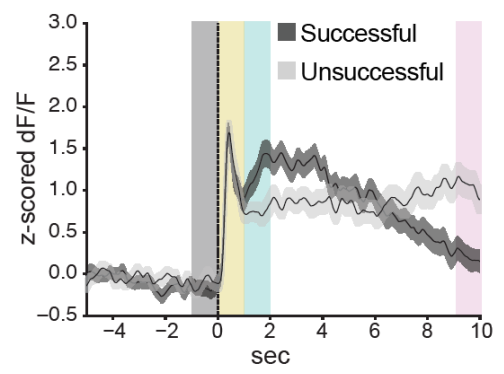

B

\section{Kajs et al. Supplemental Figure 3}

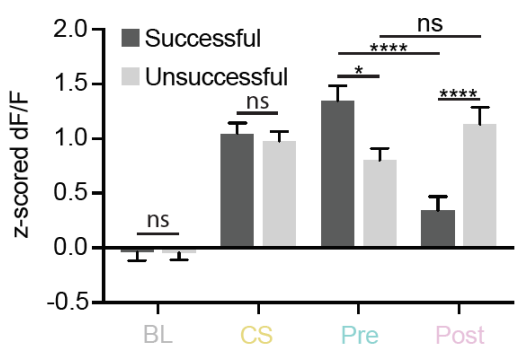

Supplemental Figure 3. Differences in dmPFC neural activity for successful versus unsuccessful trials. (A) PETH of calcium signal in dmPFC aligned to CS onset for successful (dark grey line) and unsuccessful (light grey line) trials shows differences in later parts of the trace when avoidances normally do or do not occur. Trials from Day 3 were used since equal numbers of successful and unsuccessful trials occur on this training day. Grey box, baseline period (BL); yellow box, CS response period (CS); teal box, pre avoidance period (Pre); pink box, post avoidance period (Post) (B) Quantification of the CS onset PETH shows no differences in calcium signal between successful and unsuccessful trials during the baseline period ( -1 to $0 \mathrm{~s}$ ) and the CS response period ( 0 to $1 \mathrm{~s}$ ). However, the calcium signal in the dmPFC is significantly increased during successful trials compared to unsuccessful trials during the pre avoidance period (1 to 2 s) and significantly decreased during successful trials compared to unsuccessful trials during the post avoidance period (9 to $10 \mathrm{~s}$ ) (Two-way ANOVA, Task Period $x$ Trial Type $p<0.0001$, Task Period $p<$ 0.0001 , Trial Type $p=0.5807$; Sidak's Multiple Comparisons Test, Successful Baseline vs Unsuccessful Baseline $p>0.9999$, Successful CS Response vs Unsuccessful CS Response $p=0.986$, Successful Pre Avoidance vs Unsuccessful Pre Avoidance $p=0.0022$, Successful Post Avoidance vs Unsuccessful Post Avoidance $p<0.0001 ; N=10$ mice, Successful $n=147$ trials, Unsuccessful $n=153$ trials). ns $=$ not significant, ${ }^{*} p \leq 0.05,{ }^{* * * *} p \leq 0.0001$. 
A

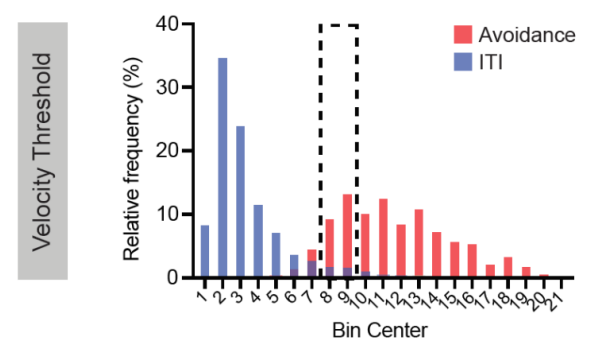

$\mathrm{D}$

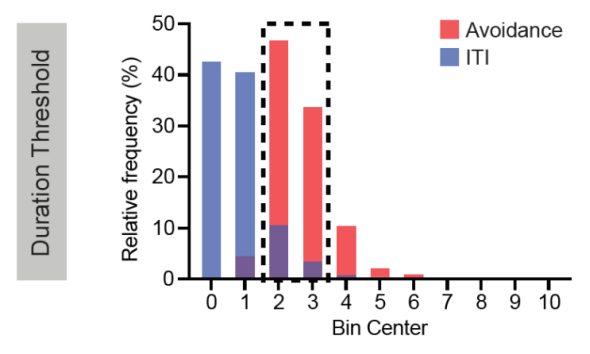

B

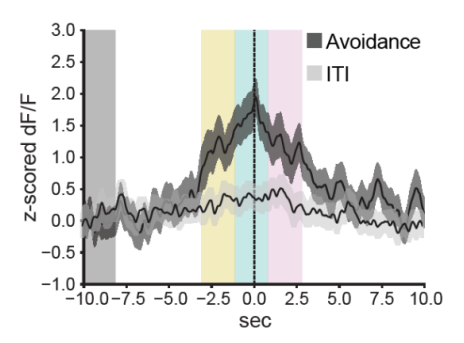

E

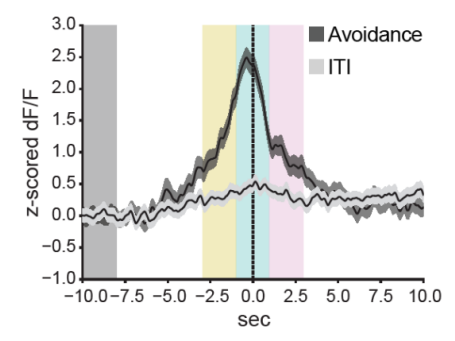

Kajs et al. Supplemental Figure 4

C

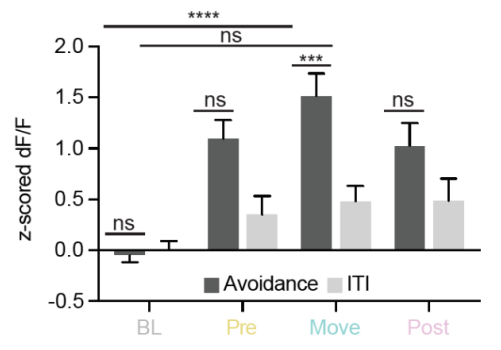

$\mathrm{F}$

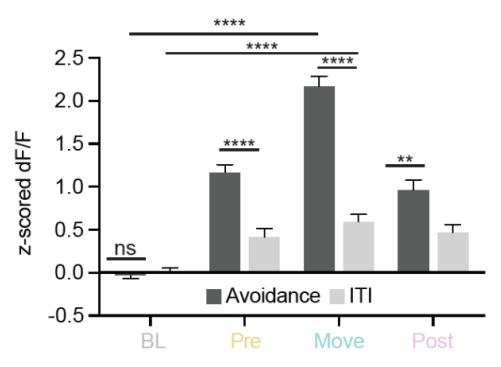

Supplemental Figure 4. Increased activity in the dmPFC during avoidance is not purely movement-related. (A) Distribution of movement velocities for intertrial (ITI) (blue) and avoidance (red) movements and their overlap (purple). (B) PETH of ITI and avoidance movements of similar velocities $(7.5 \mathrm{~cm} / \mathrm{s}$ to $9.5 \mathrm{~cm} / \mathrm{s}$ ) aligned to movement onset shows increase in calcium signal during avoidance movements that is not seen during ITI movements. Grey box, baseline period (BL); yellow box, pre movement period (Pre); teal box, movement period (Move); pink box, post movement period (Post) (C) Quantification of similar velocity movement PETH show dmPFC calcium signal is significantly increased during avoidance movements compared to ITI movements during the movement period (-1 to $1 \mathrm{~s})$, but not during baseline (-10 to $-8 \mathrm{~s})$, pre-movement (-3 to $-1 \mathrm{~s}$ ), or post-movement ( 1 to $3 \mathrm{~s}$ ) periods (Two-way ANOVA, Task Period $\mathrm{x}$ Movement Type $p=0.015$, Task Period $p<0.0001$, Movement Type $p<0.0001$; Sidak's Multiple Comparisons Test, Avoidance Baseline vs ITI Baseline $p>0.9999$, Avoidance Baseline vs Avoidance Movement $p<0.0001$, ITI Baseline vs ITI Movement $p=0.7853$, Avoidance Pre-Movement vs ITI Pre-Movement $p=0.0701$, Avoidance Movement vs ITI Movement $p=0.0009$, Avoidance Post-Movement vs ITI Post-Movement $p=$ 0.5713; $N=10$ mice, Avoidance $n=58$ trials, ITI $n=60$ trials). (D) Distribution of movement durations for ITI (blue) and avoidance (red) movements and their overlap (purple). (E) PETH of ITI and avoidance movements of similar durations ( $1.5 \mathrm{~s}$ to $3.5 \mathrm{~s}$ ) aligned to movement onset shows sharp increase in calcium signal during avoidance movements that is not seen during ITI movements. (F) Quantification of similar movement duration PETH shows dmPFC calcium signal is significantly increased during avoidance movements compared to ITI movements during pre-movement (-3 to $-1 \mathrm{~s})$, movement ( -1 to $1 \mathrm{~s})$, and postmovement ( 1 to $3 \mathrm{~s}$ ) periods, but not during the baseline (-10 to $-8 \mathrm{~s}$ ) period (Two-way ANOVA, Task Period $x$ Movement Type $p<0.0001$, Task Period $p<0.0001$, Movement Type $p<0.0001$; Sidak's Multiple Comparisons Test, Avoidance Baseline vs ITI Baseline $p>0.9999$, Avoidance Baseline vs Avoidance Movement $p<0.0001$, ITI Baseline vs ITI Movement $p<0.0001$, Avoidance Pre-Movement vs ITI PreMovement $p<0.0001$, Avoidance Movement vs ITI Movement $p<0.0001$, Avoidance Post-Movement vs ITI Post-Movement $p=0.0019 ; \mathrm{N}=10$ mice, Avoidance $\mathrm{n}=205$ trials, ITI $\mathrm{n}=227$ trials). ns $=$ not significant, ${ }^{* *} p \leq 0.01,{ }^{* * *} p \leq 0.001,{ }^{* * *} p \leq 0.0001$. 
A

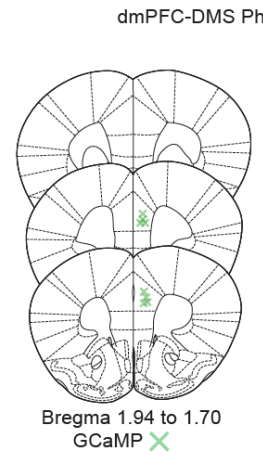

D

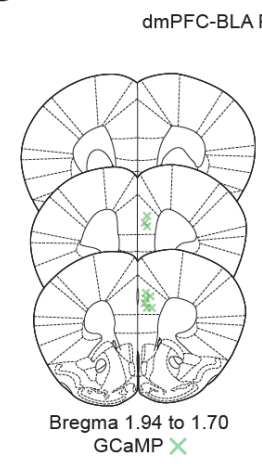

B

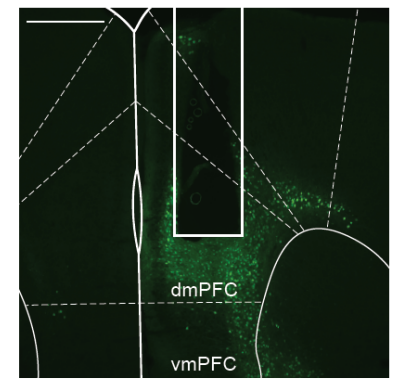

E

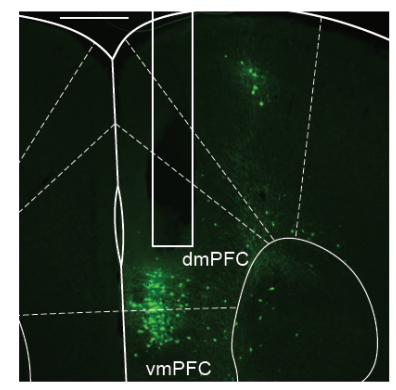

Kajs et al. Supplemental Figure 5

C

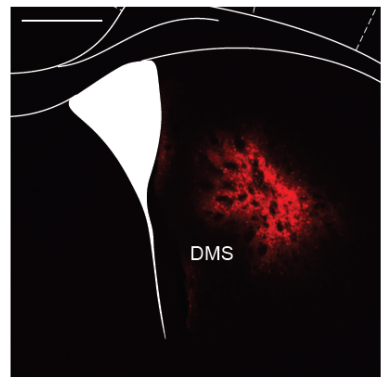

$\mathrm{F}$

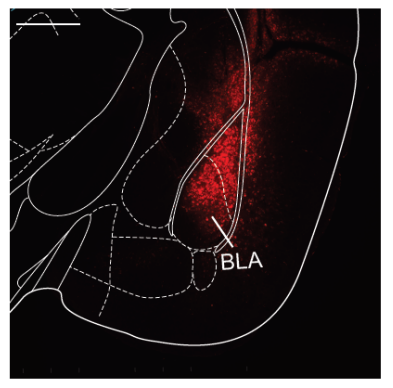

Supplemental Figure 5. Histology and targeting for dmPFC-DMS and dmPFC-BLA photometry surgeries. (A) Verification of GCaMP virus injection in dmPFC (left) and CAV2-Cre + mCherry viral injection in DMS (right) for dmPFC-DMS cohort ( $\mathrm{N}=8$ mice). (B) Representative histological image of fiber photometry implant and GCAMP viral expression in dmPFC for the dmPFC-DMS cohort. (C) Representative histological image of CAV2-Cre + mCherry viral expression in the DMS for the dmPFC-DMS cohort. (D) Verification of GCaMP virus injection in dmPFC (left) and CAV2-Cre + mCherry viral injection in BLA (right) for dmPFCBLA cohort ( $\mathrm{N}=9$ mice). (E) Representative histological image of fiber photometry implant and GCAMP viral expression in dmPFC for the dmPFC-BLA cohort. (F) Representative histological image of CAV2-Cre + mCherry viral expression in the BLA for the dmPFC-BLA cohort. Scale bar $500 \mu \mathrm{m}$. 
A

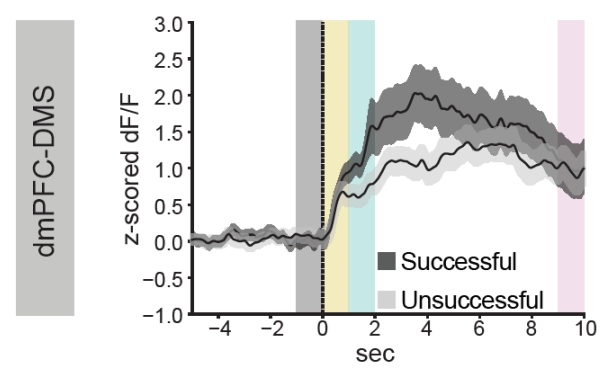

C

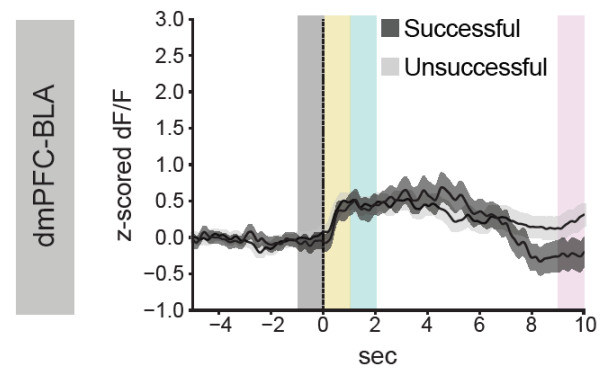

B

\section{Kajs et al. Supplemental Figure 6}
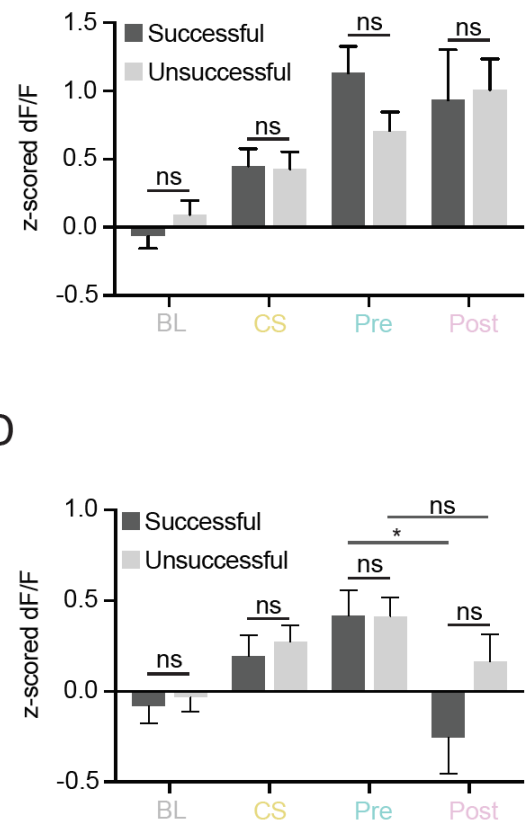

Supplemental Figure 6. No difference in dmPFC-DMS and dmPFC-BLA neural activity for successful versus unsuccessful trials. (A) PETH of calcium signal in the dmPFC-DMS projection aligned to CS onset for successful (dark grey line) and unsuccessful (light grey line) trials shows no differences between successful and unsuccessful traces. Trials from Day 3 were used since equal numbers of successful and unsuccessful trials occur on this training day. Grey box, baseline period (BL); yellow box, CS response period (CS); teal box, pre avoidance period (Pre); pink box, post avoidance period (Post) (B) Quantification of the CS onset PETH shows no differences in calcium signal between successful and unsuccessful trials during the baseline period ( -1 to $0 \mathrm{~s}$ ), CS response period ( 0 to $1 \mathrm{~s}$ ), pre avoidance period (1 to $2 \mathrm{~s}$ ), or post avoidance period (9 to $10 \mathrm{~s}$ ) for the dmPFC-DMS projection (Two-way ANOVA, Task Period $x$ Trial Type $p$ $<0.4554$, Task Period $p<0.0001$, Trial Type $p=0.7025$; Sidak's Multiple Comparisons Test, Successful Baseline vs Unsuccessful Baseline $p=0.9633$, Successful CS Response vs Unsuccessful CS Response $p>0.9999$, Successful Pre Avoidance vs Unsuccessful Pre Avoidance $p=0.4172$, Successful Post Avoidance vs Unsuccessful Post Avoidance $p=0.9978 ; N=8$ mice, Successful $n=126$ trials, Unsuccessful $\mathrm{n}=114$ trials). (C) PETH of calcium signal in the dmPFC-BLA projection aligned to CS onset for successful (dark grey line) and unsuccessful (light grey line) trials shows no differences between successful and unsuccessful traces on Day 3. (D) Quantification of the CS onset PETH shows no differences in calcium signal between successful and unsuccessful trials during the baseline period (-1 to $0 \mathrm{~s}$ ), the CS response period ( 0 to $1 \mathrm{~s}$ ), the pre avoidance period ( 1 to $2 \mathrm{~s}$ ), or the post avoidance period (9 to $10 \mathrm{~s}$ ) for the dmPFCBLA projection (Two-way ANOVA, Task Period $x$ Trial Type $p=0.3127$, Task Period $p<0.0001$, Trial Type $p=0.1204$; Sidak's Multiple Comparisons Test, Successful Baseline vs Unsuccessful Baseline $p>0.9999$, Successful CS Response vs Unsuccessful CS Response $p>0.9999$, Successful Pre Avoidance vs Unsuccessful Pre Avoidance $p>0.9999$, Successful Pre Avoidance vs Successful Post Avoidance $p=$ 0.0137, Unsuccessful Pre Avoidance vs Unsuccessful Post Avoidance $p=0.9679$, Successful Post Avoidance vs Unsuccessful Post Avoidance $p=0.3839 ; N=9$ mice, Successful $n=109$ trials, Unsuccessful $\mathrm{n}=161$ trials). $\mathrm{ns}=$ not significant, ${ }^{*} \mathrm{p} \leq 0.05$. 

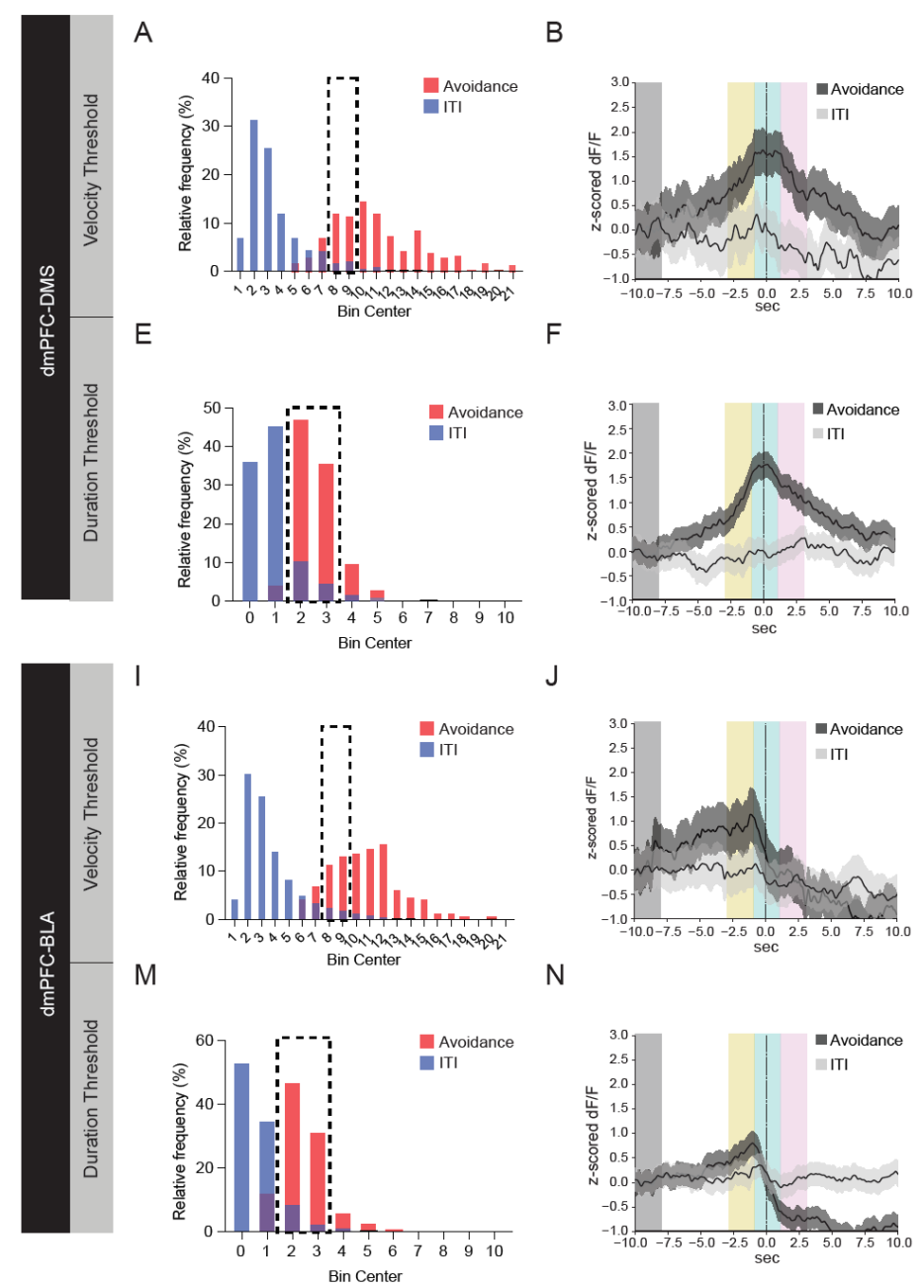

F

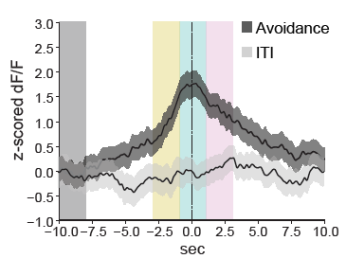

J

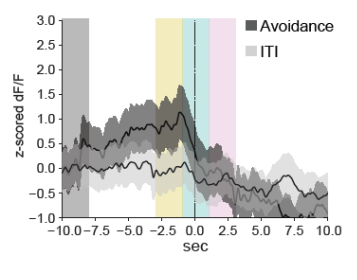

$\mathrm{N}$

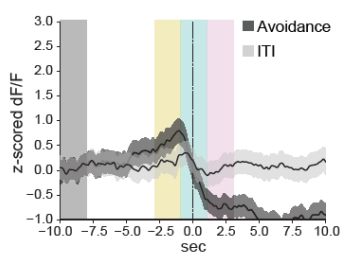

C

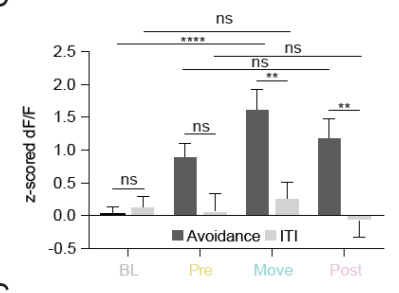

Kajs et al. Supplemental Figure 7

G

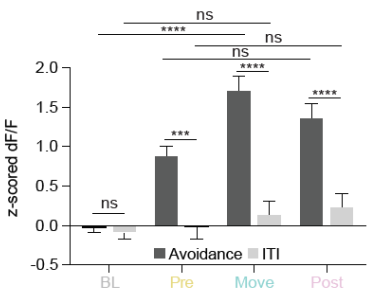

$\mathrm{H}$
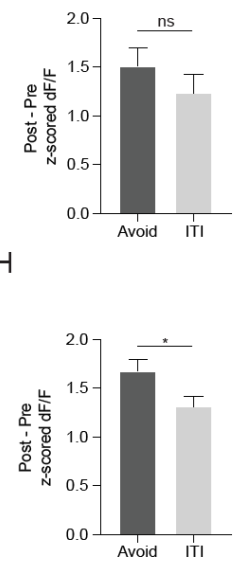

K

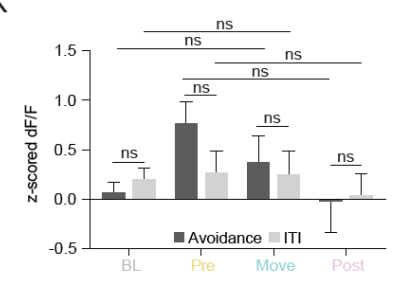

0

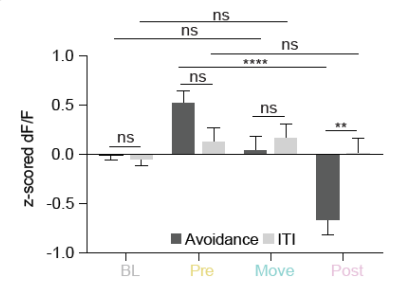

L

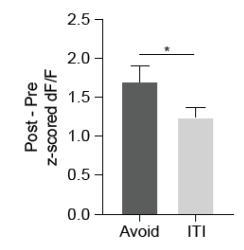

P

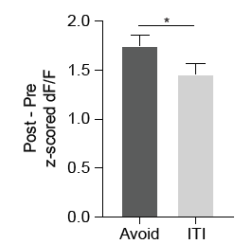

Supplemental Figure 7. Activity at avoidance onset in the dmPFC-DMS and dmPFC-BLA projections is not purely movement-related. (A) Distribution of movement velocities for ITI (blue) and avoidance (red) movements and their overlap (purple) for the dmPFC-DMS cohort. (B) PETH of ITI and avoidance movements of similar velocities $(7.5 \mathrm{~cm} / \mathrm{s}$ to $9.5 \mathrm{~cm} / \mathrm{s})$ aligned to movement onset shows increase in calcium signal during avoidance movements that is not seen during ITI movements in the dmPFC-DMS projection. Grey box, baseline period (BL); yellow box, pre movement period (Pre); teal box, movement period (Move); pink box, post movement period (Post) (C) Quantification of similar velocity movement PETH shows dmPFC-DMS calcium signal is significantly increased during avoidance movements compared to ITI movements during the movement (-1 to $1 \mathrm{~s}$ ) and post-movement (1 to $3 \mathrm{~s}$ ) periods, but not during baseline $(-10$ to $-8 \mathrm{~s}$ ) or pre-movement (-3 to $-1 \mathrm{~s})$ periods (Two-way ANOVA, Task Period $x$ Movement Type $p$ 0.0097 , Task Period p 0.0049, Movement Type $p<0.0001$; Sidak's Multiple Comparisons Test, Avoidance Baseline vs ITI Baseline $p>0.9999$, Avoidance Baseline vs Avoidance Movement $p<0.0001$, ITI Baseline vs ITI Movement $p>0.9999$, Avoidance Pre-Movement vs ITI Pre-Movement $p=0.3521$, Pre-Movement Avoidance vs Post-Movement Avoidance $p>0.9999$, Pre-Movement ITI vs Post Movement ITI $p>0.9999$, Avoidance Movement vs ITI Movement $p=0.0021$, Avoidance Post-Movement vs ITI Post-Movement $p=$ $0.0065 ; \mathrm{N}=8$ mice, Avoidance $\mathrm{n}=47$ trials, ITI $\mathrm{n}=38$ trials). (D) To compare the change in the dmPFCDMS calcium signal between pre-movement and post-movement periods for avoidance and ITI movements, we calculated the absolute value of post-movement change in calcium signal minus pre-movement change calcium signal. We find no differences in the change in dmPFC-DMS calcium signal between pre-movement and post-movement periods when comparing avoidance and ITI movements of similar velocities (Unpaired T-test $p=0.3159 ; \mathrm{N}=8$ mice, Avoidance $\mathrm{n}=47$ trials, ITI $n=38$ trials). (E) Distribution of movement durations for ITI (blue) and avoidance (red) movements and their overlap (purple) for the dmPFC-DMS 
cohort. (F) PETH of ITI and avoidance movements of similar durations (1.5 s to $3.5 \mathrm{~s}$ ) aligned to movement onset shows increase in calcium signal during avoidance movements that is not seen during ITI movements in the dmPFC-DMS projection. (G) Quantification of similar movement duration PETH shows dmPFC-DMS calcium signal is significantly increased during avoidance movements compared to ITI movements during the pre-movement ( -3 to $-1 \mathrm{~s}$ ), movement $(-1$ to $1 \mathrm{~s}$ ), and post-movement ( 1 to $3 \mathrm{~s}$ ) periods, but not during the baseline (-10 to $-8 \mathrm{~s}$ ) period (Two-way ANOVA, Task Period $x$ Movement Type $p<0.0001$, Task Period $p<0.0001$, Movement Type $p<0.0001$; Sidak's Multiple Comparisons Test, Avoidance Baseline vs ITI Baseline $p>0.9999$, Avoidance Baseline vs Avoidance Movement $p<0.0001$, ITI Baseline vs ITI Movement $p=0.9999$, Avoidance Pre-Movement vs ITI Pre-Movement $p=0.0003$, Pre-Movement Avoidance vs Post-Movement Avoidance $p=0.3365$, Pre-Movement ITI vs Post Movement ITI $p=0.9995$, Avoidance Movement vs ITI Movement $p<0.0001$, Avoidance Post-Movement vs ITI Post-Movement $p<$ $0.0001 ; \mathrm{N}=8$ mice, Avoidance $\mathrm{n}=162$ trials, ITI $n=136$ trials). $(\mathrm{H})$ The change in the dmPFC-DMS calcium signal between pre-movement and post-movement periods is significantly greater for avoidance movements compared to ITI movements of similar durations (Unpaired T-test $p=0.0166 ; \mathrm{N}=8$ mice, Avoidance $n=162$ trials, ITI $n=136$ trials). (I) Distribution of movement velocities for ITI (blue) and avoidance (red) movements and their overlap (purple) for the dmPFC-BLA cohort. (J) PETH of ITI and avoidance movements of similar velocities $(7.5 \mathrm{~cm} / \mathrm{s}$ to $9.5 \mathrm{~cm} / \mathrm{s}$ ) aligned to movement onset shows decrease in calcium signal during avoidance movements that is not seen during ITI movements in the dmPFC-BLA projection. (K) Quantification of similar velocity movement PETH shows dmPFC-BLA calcium signal is not significantly different during avoidance movements compared to ITI movements during the baseline (-10 to $8 \mathrm{~s}$ ), pre-movement (-3 to $-1 \mathrm{~s})$, movement (-1 to $1 \mathrm{~s})$, and post-movement (1 to $3 \mathrm{~s}$ ) periods (Two-way ANOVA, Task Period $x$ Movement Type $p=0.4748$, Task Period $p=0.0984$, Movement Type $p$ $=0.5066$; Sidak's Multiple Comparisons Test, Avoidance Baseline vs ITI Baseline $p>0.9999$, Avoidance Baseline vs Avoidance Movement $p>0.9999$, ITI Baseline vs ITI Movement $p>0.9999$, Avoidance PreMovement vs ITI Pre-Movement $p=0.9625$, Pre-Movement Avoidance vs Post-Movement Avoidance $p=$ 0.4489 , Pre-Movement ITI vs Post Movement ITI $p>0.9999$, Avoidance Movement vs ITI Movement $p>$ 0.9999, Avoidance Post-Movement vs ITI Post-Movement $p>0.9999 ; N=9$ mice, Avoidance $n=52$ trials, ITI $n=88$ trials) (L) The change in dmPFC-BLA calcium signal between pre-movement and post-movement periods is significantly greater for avoidance movements compared to ITI movements of similar velocities (Unpaired T-test $p=0.0487 ; \mathrm{N}=9$ mice, Avoidance $\mathrm{n}=52$ trials, ITI $n=88$ trials). (M) Distribution of movement durations for ITI (blue) and avoidance (red) movements and their overlap (purple) for the dmPFC-BLA cohort. (N) PETH of ITI and avoidance movements of similar durations (1.5 s to $3.5 \mathrm{~s}$ ) aligned to movement onset shows decrease in calcium signal during avoidance movements that is not seen during ITI movements in the dmPFC-BLA projection. (O) Quantification of similar movement duration PETH shows dmPFC-BLA calcium signal is significantly decreased during avoidance movements compared to ITI movements during the post-movement $(1$ to $3 \mathrm{~s})$ period, but not during the baseline $(-10$ to $-8 \mathrm{~s})$ premovement ( -3 to $-1 \mathrm{~s}$ ), and movement ( -1 to $1 \mathrm{~s}$ ) periods (Two-way ANOVA, Task Period $x$ Movement Type $p=0.0001$, Task Period $p<0.0001$, Movement Type $p=0.2688$; Sidak's Multiple Comparisons Test, Avoidance Baseline vs ITI Baseline $p>0.9999$, Avoidance Baseline vs Avoidance Movement $p>0.9999$, ITI Baseline vs ITI Movement $p=0.9935$, Avoidance Pre-Movement vs ITI Pre-Movement $p=0.4972$, PreMovement Avoidance vs Post-Movement Avoidance $p<0.0001$, Pre-Movement ITI vs Post Movement ITI $p>0.9999$, Avoidance Movement vs ITI Movement $p>0.9999$, Avoidance Post-Movement vs ITI PostMovement $p=0.0017 ; \mathrm{N}=9$ mice, Avoidance $n=165$ trials, ITI $n=211$ trials). (P) The change in dmPFCBLA calcium signal between pre-movement and post-movement periods is significantly greater for avoidance movements compared to ITI movements of similar durations (Unpaired T-test $p=0.0473 ; \mathrm{N}=9$ mice, Avoidance $n=165$ trials, ITI $n=208$ trials). $n s=$ not significant, ${ }^{*} p \leq 0.05,{ }^{* *} p \leq 0.01,{ }^{* * *} p \leq 0.001$, ${ }^{* * * *} p \leq 0.0001$. 